bislang wenig untersuchten gewalthaltigen Computerspiele. Damit ist es Anliegen dieser Arbeit, den Erklärungswert zentraler kognitiver und affektiver Minderungshypothesen für den aufgefundenen negativen Zusammenhang zwischen Unterhaltungsmediennutzung und Schulleistung am Beispiel der Nutzung gewalthaltiger Computerspiele auf ihre Relevanz hin zu überprüfen und zur Theoriebildung in diesem Feld beizutragen.

Im folgenden Kapitel wird der Frage nachgegangen werden, welche Kenntnisse bislang zu den kognitiven und affektiven Wirkungen von gewalthaltigen Computerspielen vorliegen.

\title{
1.3 Gewalthaltige Computerspiele: Kognitives und emotionales Wirkpotential
}

\subsubsection{Computerspielen und kognitive Beanspruchung}

Computerspielen unterscheidet sich hinsichtlich der beteiligten Sinnesmodalitäten kaum vom klassischen Fernsehen: auch hier werden akustische und visuelle Informationen über technische Ausgabemedien vermittelt und bestimmen auf diese Weise das Rezeptionsgeschehen. Hinsichtlich der beanspruchten kognitiven Leistungen bestehen dennoch deutliche Unterschiede: Als interaktives Medium wird das Rezeptionsgeschehen beim Computerspiel durch die Eingaben des Nutzers und die Folgen dieser Eingaben im Spiel bestimmt, die einen wechselseitig-reaktiven Kreislauf bilden (vgl. Klimmt, 2004, S. 3). Computerspiele erfordern deshalb im Vergleich zu Filmen, die auch neben anderen Aktivitäten unter geringer aktiver Zuwendung genutzt werden können, ein beständiges Maß an Aufmerksamkeit (Kunczik \& Zipfel, 2004, S. 201).

Dabei kommt es auf das verwendete Computerspiel an, welche Leistungen die Spieler erbringen müssen, um die spielimmanenten Ziele zu erreichen. Der Computerspielforscher Klimmt (2004, S. 5) schlägt vor, Computerspiele insbesondere danach zu unterscheiden, ob sie eher Komplexität oder eher Geschwindigkeit in den Vordergrund stellen: Spiele, die eher Komplexität in den Vordergrund stellen, erfordern vom Spieler statt schneller Spielhandlungen vorzugsweise die Berücksichtigung und Überwachung einer Vielzahl von sich fortlaufend verändernden Informationen und die Umsetzung dieser Informationen in strategische Überlegungen. Typische Beispiele für derartige Spiele sind Denkspiele, Strategiespiele und Wirtschafts- bzw. Aufbausimulationen. Stattdessen legen Spiele, die eher Spielgeschwindigkeit in den Vordergrund stellen, den Akzent auf schnelle Reaktionen, die Erfassung und geschickte Ausnutzung räumlich-visueller Zusammenhänge und die schnelle Koordination komplexer Steuerbefehle. Gewalthaltige 
Computerspiele (Shooter und Kampfspiele) sind nahezu ausschließlich der zweiten Kategorie zugehörig. ${ }^{29}$ Sie erfordern in aller Regel schnelle Reaktionen, weisen bedrohliche Handlungsszenarien auf ("Kampf auf Leben und Tod") und setzen den Spieler unter einen vergleichsweise hohen Handlungsdruck. So gut wie alle Spielgenre können inzwischen sowohl offline (ohne aktive Internetverbindung) als auch online (im Internet) gespielt werden. Onlinespiele ermöglichen dabei häufig glaubwürdigere und komplexere Interaktionen, indem die Handlungen des Spielers nicht ausschließlich durch regelgeleitete programmierte Routinen beantwortet werden. Dadurch werden Handlungsabläufe weniger vorhersehbar. Zudem können die Spieler in Onlinespielen miteinander kommunizieren und sich in Spielergruppen (Clans, Gilden) zusammenschließen.

\subsubsection{Forschungsstand: Computerspielnutzung und kognitive Leistungsparameter}

"Although the visual capabilities of television, video games, and the Internet may develop impressive visual intelligence, the cost seems to be deep processing: mindful knowledge acquisition, inductive analysis, critical thinking, imagination, and reflection. It is difficult for schools to teach reflective habits of mind to children whose informal education and cognitive socialization have not prepared them for this kind of learning and thinking" (Greenfield, 2009, S. 71).

Angesichts des aufgezeigten negativen Zusammenhangs zwischen Computerspielnutzung und Schulleistung mag es überraschen, dass die empirische Datenlage insgesamt auf eine Vielzahl von positiven Wirkungen von Computerspielen auf kognitive Fertigkeiten hinweist. Forschungsdaten zu kognitiven Auswirkungen von Computerspielnutzung wurden bislang nahezu ausschließlich im Rahmen von laborexperimentellen Zugängen gewonnen. Hierbei lassen sich experimentelle und quasiexperimentelle Zugangswege unterscheiden: Während in quasiexperimentellen Studien in der Regel kognitive Leistungsparameter von Versuchspersonen mit unterschiedlichem Nutzungsgewohnheiten miteinander verglichen werden, wird im Rahmen von experimentellen Untersuchungen die Wirkung bestimmter Computerspieltreatments auf nachfolgende kognitive Leistungen untersucht (vgl. An-

29 Eine Ausnahme stellen militärisch-kriegerische Interaktionen in Strategiespielen dar, die zum Teil auch deutlich visualisiert werden. Dies ist jedoch häufig gerade in actionreichen Echtzeit- und weniger in rundenbasierten Strategiespielen der Fall. 
drews \& Murphy, 2006). Die hierbei untersuchten Leistungen ${ }^{30}$ lassen sich maßgeblich dem räumlich-visuellen Verarbeitungsspektrum zuordnen (Subrahmanyam, Greenfield, Kraut, \& Gross, 2001).

Im Rahmen quasiexperimenteller Untersuchungen konnte gezeigt werden, dass erfahrene Computerspieler gegenüber weniger Erfahrenen oder Nichtspielern höhere visuelle Gedächtnisleistungen erbringen (Boot, Kramer, Simons, Fabiani, \& Gratton, 2008; Ferguson, Cruz, \& Rueda, 2008), schnell bewegende Objekte besser verfolgen können (Boot, et al., 2008), schneller zwischen verschiedenen Aufgaben wechseln können (Andrews \& Murphy, 2006; Boot, et al., 2008), in mentalen Rotationsaufgaben ${ }^{31}$ besser abschneiden (Boot, et al., 2008), höhere Leistungen hinsichtlich verschiedener Funktionsbereiche der selektiven Aufmerksamkeit aufweisen (Castel, Pratt, \& Drummond, 2005; Dye, Green, \& Bavelier, 2009; Green \& Bavelier, 2003; Greenfield, Dewinstanley, Kilpatrick, \& Kaye, 1994) und über eine bessere Hand-Augen-Koordination verfügen (Rosser, et al., 2007). Zudem kann davon ausgegangen werden, dass gerade Nutzer von Denk- und Strategiespielen bestimmte Problemlösefähigkeiten erwerben bzw. ausbauen können, die sie auf ähnliche Problemlösekontexte (insbesondere in anderen Spielen) übertragen können (Kraam-Aulenbach, 2000).

Diese quasiexperimentellen Befunde konnten in experimentellen Trainingsstudien weitestgehend bestätigt werden. Greenfield und Kollegen (1994) wiesen nach einem fünfstündigen Training mit dem Action-Arcade-Spiel Robotron zwar keine ganzheitliche Verbesserung der visuellen Aufmerksamkeit, jedoch zuverlässigere Reaktionen auf visuelle Reize mit geringer Auftretenswahrscheinlichkeit nach. Green und Bavelier (2003) konnten zeigen, dass sowohl bei Nichtspielern wie auch erfahrenen Spielern mittels eines zehntägigen Trainings mit einem First-PersonShooter (Medal of Honor) selektive Aufmerksamkeitsleistungen verbessert werden können (Green \& Bavelier, 2003). In einer weiteren aktuellen Studie konnte gezeigt werden, dass ein 10-stündiges Training mit einem First-Person-Shooter (Medal of Honor) bei Frauen und Männern ohne Erfahrungen mit actionreichen Computerspielen zu einer gesteigerten visuellen Aufmerksamkeitsleistung führt (Spence, Yu, Feng, \& Marshman, 2009).

Auch zum räumlichen Vorstellungsvermögen (Visualization) konnten positive Effekte von Computerspieltreatments nachgewiesen werden. Subrahmanyam und

30 Die Recherche wurde über die Datenbanken PubMed, PsyCONTENT und Google Schoolar durchgeführt. Es wurden deutsche und englische Suchbegriffe eingesetzt. Dabei wurde die UV-Ebene computerspiel, videospiel, computer game, video game mit den Begriffen attention, concentration, cognition, memory, aufmerksamkeit, kognition, gedächtnis der AVEbene kombiniert. Zusätzlich wurden im "Schneeballverfahren" Studien anhand vorhandener Quellennachweise einbezogen.

31 Mentale Rotation wird als Fähigkeit verstanden, das Erscheinungsbild zwei- oder dreidimensionaler Figuren vorherzusehen, wenn diese um einen bestimmten Grad um eine bestimmte Achse gedreht werden (vgl. De Lisi \& Wolford, 2002, S. 272). 
Greenfield konnten so etwa zeigen, dass das Spielen (3 x 45 Minuten) von Marple Madness - einem Geschicklichkeitsspiel bei dem eine Kugel über einen dreidimensionalen Parkur navigiert werden muss - das räumliche Vorstellungsvermögen gegenüber einem einfachen Wortspiel erhöht (Subrahmanyam \& Greenfield, 1994). De Lisi und Wolford (2002) konnten an Kindern im Alter zwischen 8 und 9 Jahren Leistungssteigerungen in einem mentalen Rotationstest nach einem Computerspieltreatment bestehend aus 11 jeweils 30-minütigen Einzelsessions des Denkspiels Tetris gegenüber einem einfachen Wortspiel nachweisen. Während die Jungen vor dem Treatment höhere Leistungen als die Mädchen aufwiesen, bestanden innerhalb der Treatmentgruppe in der Postmessung keine geschlechtsspezifischen Unterschiede in der Fähigkeit zur mentalen Rotation (De Lisi \& Wolford, 2002). Dies konnte in ähnlicher Weise für erwachsene Probanden nach nur zwei 30-minütigen Spielsessions mit einem Tetris-ähnlichen Spiel (Blockout) gegenüber einem Computerkartenspiel (Solitaire) gezeigt werden (De Lisi \& Cammarano, 1996). In einem Experiment mit $\mathrm{N}=110$ Studenten verglichen Feng und Kollegen die Auswirkungen eines 10-stündigen Trainings mit einem First-Person-Shooter (Medal of Honor) und einem Geschicklichkeitsspiel (Ballance) auf die visuelle Aufmerksamkeit und Fähigkeit zur Mentalen Rotation von N = 20 Studenten (Feng, Spence, \& Pratt, 2007). Während das grafisch einfach gestaltete Geschicklichkeitsspiel zu keiner Verbesserung in beiden Leistungstests führte, ergab sich eine Leistungssteigerung für die Spieler des First-Person-Shooters. Die weiblichen Teilnehmer profitierten insgesamt stärker, wodurch sich hinsichtlich der visuellen Aufmerksamkeitsleistung kein Geschlechtsunterschied in der Postmessung ergab, der noch in der Prämessung bestanden hatte. Auch hinsichtlich des Mentalen Rotationstests wurden die Leistungsunterschiede zwischen den Geschlechtern geringer (Feng, et al., 2007). Insbesondere Green und Bavelier konnten in vielfältigen Experimenten nachweisen, dass First-Person-Shooter mit ihrer elaborierteren grafischen Gestaltung räumlich-visuelle Fähigkeiten stärker befördern können als grafisch einfacher gestaltete Geschicklichkeitsspiele (Green \& Bavelier, 2006 a, 2006 b, 2007). Es kann vermutet werden, dass sich mit höherem Reifegrad in der Darstellung im Zuge der technischen Entwicklung auch größere Effekte auf räumliche-visuelle Verarbeitungsleistungen ergeben könnten (Subrahmanyam, Greenfield, et al., 2001, S. 13).

Auch hinsichtlich der Hand-Augen-Koordination konnten positive Effekte von Computerspieltreatments nachgewiesen werden. In einer Studie wurde untersucht, inwieweit sich die Nutzung eines visuell anspruchsvollen Computerspiels (First Person Shooter: Half Life) im Vergleich zu einem Denkspiel (Schachspiel: Chessmaster) und einer Kontrollbedingung (kein Computerspieltraining) auf die Testleistungen von Medizinstudenten in zwei Endoskopie-Untersuchungssimulationen auswirkt (Schlickum, Hedman, Enochsson, Kjellin, \& Felländer-Tsai, 2009). Das 
Computerspieltraining wurde an insgesamt 25 Tagen für jeweils 30 bis 60 Minuten durchgeführt. Es zeigte sich, dass beide Computerspieltreatments zu signifikant besseren Testleistungen in der ersten Simulation (MIST-VR) und nur das visuellanspruchsvolle Computerspiel zu signifikant besseren Leistungen in der zweiten Simulation (GI Mentor II) führte.

Neben Studien zum räumlich-visuellen Verarbeitungsspektrum gibt es nur sehr wenige Untersuchungen zu den Auswirkungen von Computerspielen auf kognitive Leistungsaspekte, die in Computerspielen nicht unmittelbar trainiert werden oder breitere Funktionseinheiten betreffen (vgl. Tabelle 2). In einer experimentellen Untersuchung von Skosnik, Chatterton, Swisher und Park (2000) wurde die Wirkung eines gewalthaltigen Computerspiels auf die selektive Aufmerksamkeit im Rahmen eines Negativ-Priming-Paradigmas untersucht. Diese Studie geht insoweit über die bestehende Datenlage zu Computerspielen und Aufmerksamkeitsleistung hinaus, dass in dieser Untersuchung gewalthaltige Computerspiele als Stressoren aufgefasst wurden, welche die nachfolgende selektive Aufmerksamkeit zu relevanten Reizen unter Ausblendung irrelevanter Reize negativ beeinflussen könnten. Die Ergebnisse zeigen, dass nach dem 15-minütigen Spielen eines gewalthaltigen Computerspiels die Versuchspersonen zwar schneller auf Zielreize reagierten, gleichzeitig aber weniger gut dazu in der Lage waren, irrelevante Reize zu unterdrücken. Die Beeinträchtigung in der Unterdrückung irrelevanter Reize fiel besonders schlecht bei Personen aus, die mit einer erhöhten Cortisolausschüttung auf das Spiel reagierten.

Barlett und Kollegen (2009) setzten einen computergestützten Leistungstest (SynWin) ein, bei dem simultan vier verschiedene kognitive Aufgaben zu bearbeiten sind, die jeweils auf einem separaten Quadranten des Bildschirms dargestellt werden. Dabei kamen neben visueller selektiver Aufmerksamkeit (Überwachung eines absinkenden Treibstofflevels in einem Benzintank) und akustischer selektiver Aufmerksamkeit (Reaktion auf hohe Töne mit Mausklick, ignorieren tiefer Töne) mit dem Sternberg-Task ${ }^{32}$ und dem Aufaddieren dreistelliger Ziffern auch arbeitsgedächtnisnahe Aufgaben zum Einsatz. Da jedoch alle Aufgaben parallel eingesetzt wurden und ihre Bewältigung in einen gemeinsamen Summenscore eingingen, können die kognitiven Leistungsaspekte nicht separat betrachtet werden. In einer ersten quasiexperimentellen Studie an N $=37$ Studenten zeigte sich, dass die Testleistungen in diesem Verfahren nicht mit Computerspielerfahrungen der Probanden in Zusammenhang standen. In einer zweiten experimentellen Studie an $\mathrm{N}=113$ Studenten zeigte sich jedoch, dass sich sowohl ein Training mit einem gewalthaltigen als auch mit einem gewaltneutralen Computerspiel positiv auf die Leistungen

32 Beim Sternberg-Task mussten die Probanden sechs Buchstaben überwachen und dann bei Ausblendung des Displays schnell entscheiden, ob ein weiterer präsentierter Buchstabe in der Präsentationsreihe enthalten war (Barlett, Vowels, et al., 2009, S. 98). 
in SynWin gegenüber einer Kontrollbedingung (Internetrecherche) auswirkte. Als gewalthaltiges Spiel kam hierbei das Strategiespiel Red Alert 2 zum Einsatz, welches jedoch allenfalls moderate Gewaltdarstellungen enthält.

Boot und Kollegen führten neben einer quasiexperimentellen Studie an N = 21 Nicht- und Vielspielern auch eine experimentelle Untersuchung an $\mathrm{N}=82$ Nichtspielern durch, die im Rahmen von 15 Trainingseinheiten innerhalb eines 5-Wochen Zeitraums entweder ein Denk-, Strategie- oder Shooterspiel spielten oder einer Kontrollgruppe ohne spezifisches Computerspieltraining zugewiesen wurden (Boot, et al., 2008). Dabei wurde eine große Anzahl von Testverfahren eingesetzt, die neben räumlich-visuellen Fähigkeiten auch Instrumente zur Messung von Arbeitsgedächtnisleistungen bzw. exekutiven Funktionen (Tower Of London, Working Memory Span, Raven Matrices) umfasste. Die Autoren erwarteten je nach Computerspiel und der darin zur Anwendung gebrachten Leistungen die Förderung bestimmter kognitiver Fähigkeiten. Jedoch zeigte sich lediglich hinsichtlich des Untertests Mentale Rotation eine Leistungssteigerung in den Computerspielbedingungen gegenüber der Kontrollbedingung. Hier profitierten die Probanden am meisten, die der Denkspielbedingung (Tetris) zugewiesen wurden. Alle anderen abhängigen Variablen wurden durch das Training weder positiv noch negativ beeinflusst (Boot, et al., 2008).

Reinecke und Trepte (2008) konnten in einer quasiexperimentellen Studie an $\mathrm{N}=129$ Studenten zeigen, dass die Probanden nach einer fünfminütigen Nutzung des Denkspiels Tetris ein höheres Arousal und eine höhere Konzentrationsfähigkeit aufwiesen als Probanden einer Wartebedingung. Eine Besonderheit dieser Untersuchung gegenüber anderen Studien stellt dar, dass die Konzentrationsmessung mithilfe des KLT-R (Konzentrations-Leistungs-Test: Revidierte Fassung) von Mayrhofer (2004) operationalisiert wurde. In diesem Verfahren müssen die Probanden unter Zeitdruck einfache Rechenaufgaben lösen und dabei Zwischenergebnisse sowie Rechenregeln zum Umgang mit den Zwischenergebnissen im Arbeitsgedächtnis verfügbar halten. Damit bildet dieses Verfahren in weit geringerem Maße als andere Testverfahren visuelle Aufmerksamkeitsleistungen und in höherem Maße Konzentrationsfähigkeit im Sinne von Belastbarkeit, Ausdauer und Ermüdungsresistenz ab (Mayrhofer, 2004). Da jedoch die Probanden selbst entscheiden konnten, ob sie der Computerspiel- oder Wartebedingung zugewiesen werden wollten, kann nicht eingeschätzt werden, inwieweit das Computerspieltreatment tatsächlich für die erhöhte Konzentrationsleistung verantwortlich war oder ob sich Probanden mit höherer Konzentrationsleistung auch freiwillig lieber den Spielen zugewendet haben (z. B. weil sie munterer waren).

Dworak und Kollegen (Dworak, Schierl, Bruns, \& Struder, 2007) untersuchten die Auswirkungen von Computerspiel- und Fernsehnutzung auf die visuelle und verbale Gedächtnisleistung innerhalb eines experimentellen Within-Subject-De- 
signs. Hierzu wurden die kindlichen Versuchspersonen an drei Versuchstagen vor dem Einschlafen jeweils einer anderen Abendbedingung ausgesetzt: Nutzung eines actionreichen Rennspiels, Nutzung eines spannenden Films oder freie nicht-mediale Aktivitäten (Kontrollbedingung). Hinsichtlich der vorab im Rahmen des VVM (Visueller und Verbaler Merkfähigkeitstest) erlernten visuellen und verbalen Informationen zeigte sich nur für die verbale Gedächtnisleistung eine signifikant erhöhte Vergessensrate in der Computerspielbedingung vs. Kontrollbedingung. Hinsichtlich der visuellen Gedächtnisleistung ergaben sich keine Gruppenunterschiede. Die Autoren erklären ihren Befund mit kurzfristigen emotionalen Einflüssen der Rezeptionssituation auf die Gedächtniskonsolidierung und sehen hierin im Sinne der Gedächtnis-Defizit-Hypothese (vgl. Kapitel 1.2.3) eine mögliche Erklärung für den negativen Zusammenhang zwischen Computerspielnutzung und verringerten Schul- und Leseleistungen: "Finally, our results could provide a plausible explanation concerning the effects of media exposure on poor school completion and especially poor reading skills, derived from the results of the VVM" (Dworak, et al., 2007, S. 984). Dennoch muss die geringe Stichprobengröße von $\mathrm{N}=11$ auch innerhalb eines Within-Subject-Designs Anlass zur Vorsicht bei der Ergebnisinterpretation geben.

Eine letzte hier noch zu berichtende Studie stellt die experimentelle Untersuchung von Maass, Klöpper, Michel und Lohaus (2009) dar. In dieser Untersuchung an $\mathrm{N}=117$ Studenten wurden sowohl psychometrische Aufmerksamkeitsdiagnostik als auch verbale Gedächtnisleistung berücksichtigt. Als Treatmentbedingungen kamen gewaltneutrale und gewalthaltige Computerspiele und Filme zum Einsatz. Dabei zeigte sich ein Trend zu einer im Anschluss an das 30-minütige Treatment verringerten Gedächtnisleistung, die jedoch nicht statistisch signifikant wurde, und eine signifikant geringere Aufmerksamkeitsleistung in den Gewaltbedingungen (Maass, et al., 2009).

Insgesamt zeigt sich anhand des Forschungsüberblicks, dass insbesondere Kenntnisse zu positiven kognitiven Wirkungen von Computerspielen vorliegen. Damit scheint die Befundlage zunächst in einem gewissen Widerspruch zur Schulleistungsforschung zu stehen, die in der Tendenz negative Zusammenhänge zwischen Computerspielnutzung und Schulleistung aufzeigt. Welche Erklärung bietet sich hierfür an? Zum einen kann argumentiert werden, dass sich die meisten bislang vorliegenden Studien auf kognitive Leistungsindikatoren konzentrieren, die in Computerspielen unmittelbar trainiert werden. Nahezu alle aufgefundenen Studien (mit Ausnahme von Dworak, et al., 2007; Maass, et al., 2009; Skosnik, et al., 2000) sind ihrem immanenten Verständnis nach "Trainingsstudien" und widmen sich der Frage, inwieweit während des Computerspielens erbrachte mentale Leistungen tatsächlich verbessert werden. In diesem Zusammenhang erscheint es plausibel, dass Computerspieler in besonderer Weise lernen, komplexe visuelle Informatio- 
nen zu verarbeiten, schnell auf Zielreize zu reagieren und diese in geeignete motorische (Hand-)Bewegungen zu übersetzen. Damit kann dem aktuellen Forschungsstand zur Computerspielnutzung und Kognition bislang in erster Linie entnommen werden, dass mit Computerspielen bestimmte Fertigkeiten trainiert werden können. ${ }^{33}$ Ein umfassendes Verständnis der Wirkungen von Computerspielen auf die kognitive Leistungsfähigkeit kann hieraus jedoch bislang kaum abgeleitet werden. Eine wichtige Frage stellt sich zum einen dahingehend, ob Transfereffekte wirksam werden, indem die im Rahmen der Computerspielnutzung erworbenen Fähigkeiten auch im wirklichen Leben zur Anwendung gebracht werden können. Dies ist bislang jedoch kaum erforscht worden:

"However, there is no research that actually documents a link between video game playing, attentional skills, and success in academic performance or specific occupations" (Subrahmanyam, Kraut, et al., 2001, S. 15).

Zwar zeigt sich am Beispiel der Hand-Augen-Koordination, dass erfahrene Computerspieler durchaus bestimmte Vorteile in spezifischen beruflichen Kontexten (z. B. Chirurg) haben könnten. Auf Basis der vorhandenen Daten, gerade in Hinblick auf die negativen Zusammenhänge zwischen Computerspielnutzung und Schulleistung, haben derartige Überlegungen jedoch höchst spekulativen Charakter. Um zu einem umfassenderen Verständnis darüber zu gelangen, wie die Nutzung von Computerspielen kognitive Leistungen im realen Leben beeinflusst, muss für die zukünftige Forschung ein weiteres und nicht nur auf den visuellen Bereich beschränktes Verständnis von Kognition als notwendig erachtet werden:

"It is speculated that differences between the video games used and measures of spatial abilities attribute to this discrepancy, which is the reason why general cognition is the primary focus of the current research. We believe that before specific cognitive variables should be measured, general cognition should be the focus (...)" (Barlett, Vowels, et al., 2009, S. 97).

Ansonsten könnte bei einer Fokussierung auf räumlich-visuelle Verarbeitungsleistungen die Entwicklung anderer kognitiver Fähigkeiten vernachlässigt werden:

"Although visual literacy is a tool in scientific thinking and can lead to discoveries (...) scientific thinking goes beyond the techniques provided by visual literacy, highlighting the importance of a number of other qualities: reflection, inductive analysis, critical thinking, mindful thought, and imagination" (Greenfield, 2009, S. 71).

33 Ein besonderer Bereich, in dem Computerspiele zu Trainingszwecken eingesetzt werden, ist militärisches Training. So weisen Buckley und Anderson (2006) darauf hin, dass die amerikanische Armee hohe Geldbeträge in Computerspieltrainingssimulationen investiert, die in der Ausbildung militärischen Personals eingesetzt werden, und mit denen beispielsweise operative und strategische Abläufe von Kampfeinsätzen trainiert werden. 
Neben der Notwendigkeit eines globaleren Verständnisses kognitiver Fähigkeiten kann nach wie vor kaum eingeschätzt werden, ob die positiven oder negativen Einflüsse von Computerspielen, insbesondere von gewalthaltigen Spielen, auf kognitive Prozesse insgesamt überwiegen (vgl. Greenfield, 2009, S. 71). Viele Studien haben bislang gewaltneutrales Stimulusmaterial wie Denk-, Puzzle- und Geschicklichkeitsspiele verwendet (Barlett, Vowels, et al., 2009, S. 97). Zwar wird anhand der vorhandenen Studien deutlich, dass einige gewalthaltige Computerspiele hinsichtlich räumlich-visueller Leistungen sogar ein in besonderer Weise förderliches Potential aufweisen (Barlett, Vowels, et al., 2009). Dies erscheint jedoch insoweit erwartungskonform, als dass gerade Shooterspiele eine besonders elaborierte Grafik sowie hochwertige Abbildungen räumlicher Zusammenhänge aufweisen. Ein systematischer Vergleich von grafisch fortgeschrittenen gewalthaltigen und gewaltneutralen Spielen hat noch nicht stattgefunden. Inwieweit sich gewalthaltige und gewaltneutrale Computerspiele in unterschiedlicher Weise auf breitere kognitive Leistungsparameter auswirken, kann bislang anhand der vorhandenen Datenlage nicht eingeschätzt werden (Barlett, Vowels, et al., 2009, S. 97).

Um die Betrachtung der kognitiven Auswirkungen von Computerspielen zu vervollständigen, soll im nächsten Kapitel die Frage behandelt werden, welche spezielleren Kenntnisse zu kurzfristigen emotionalen Auswirkungen von gewalthaltigen Computerspielen vorliegen. 


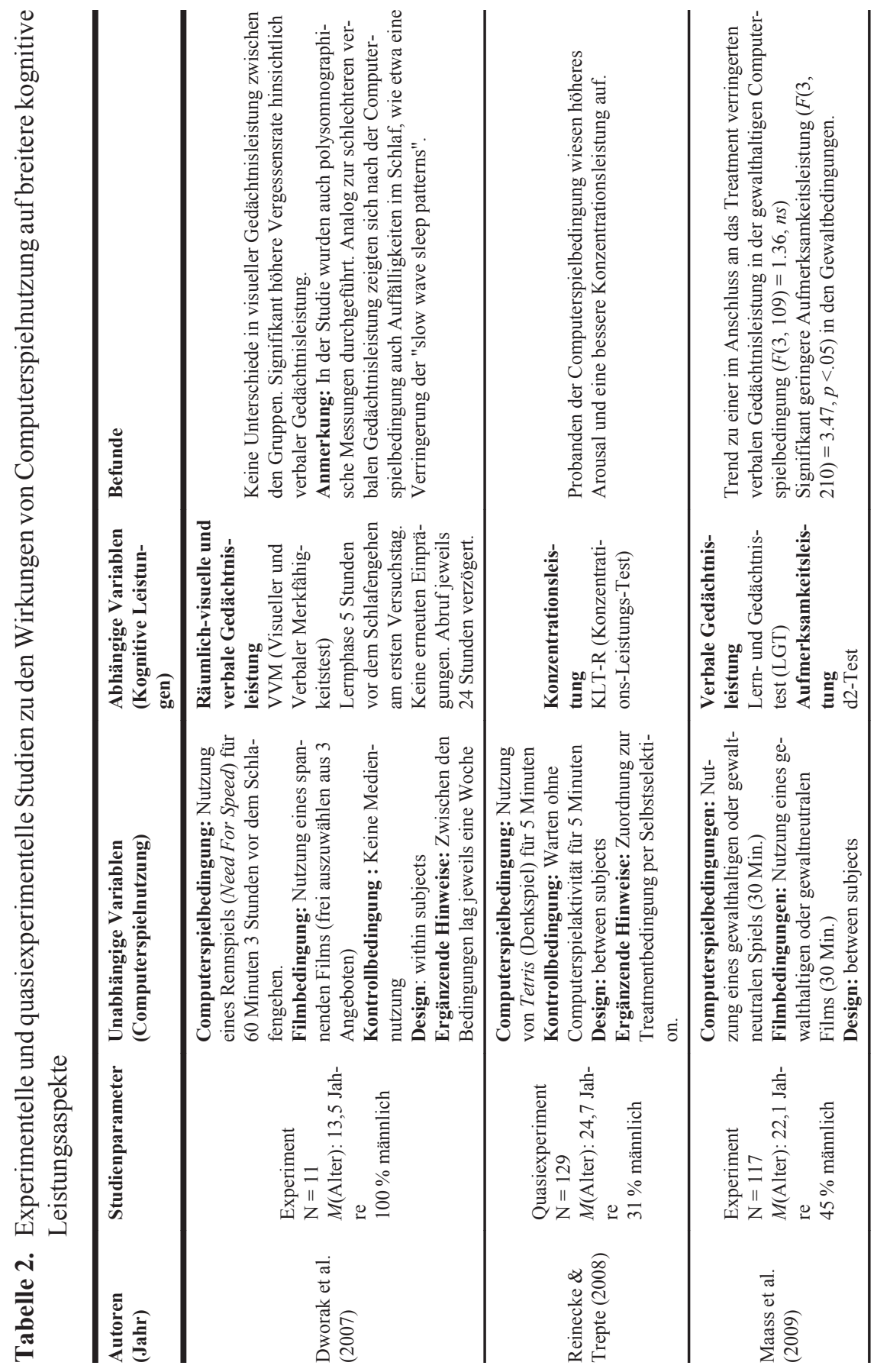




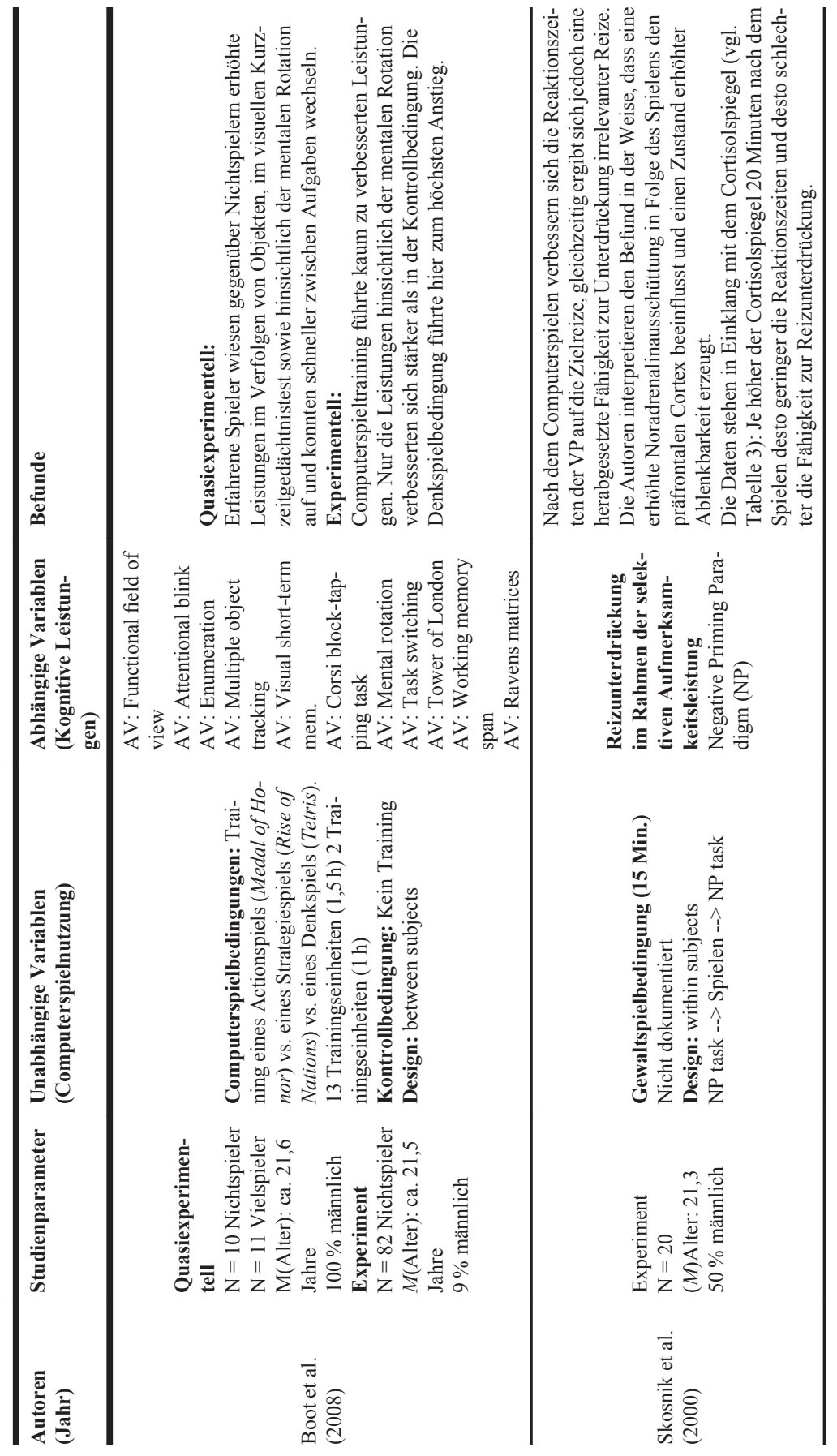




\subsubsection{Kurzfristige emotionale Wirkungen gewalthaltiger Computerspiele}

Die Forschung zu den affektiven Wirkungen von gewalthaltigen Computerspielen ist vorrangig durch die Untersuchung aggressionsnaher Verhaltenskonstrukte geprägt, die deshalb den Ausgangspunkt dieses Kapitels bilden sollen. Dieser Forschungsbereich lässt sich auf zwei zentrale Fragestellungen eingrenzen (vgl. C. A. Anderson \& Bushman, 2001, S. 356):

1. Besteht ein Zusammenhang zwischen der Nutzung gewalthaltiger Spiele und Aggressivität?

2. Wie kann ein möglicher Zusammenhang zwischen der Nutzung gewalthaltiger Spiele und Aggressivität theoretisch erklärt werden?

Als besonders einflussreicher Ansatz zur systematischen Erforschung und Einordnung dieser Fragestellungen gilt das General Aggression Model (GAM), ${ }^{34}$ welches zentrale Lern-, Skript-, Priming-, Erregungsübertragungs- und Desensibilisierungstheorien in ein allgemeines Mehr-Phasen-Modell zur Erklärung von Aggressivität integriert. ${ }^{35}$ Das GAM stützt sich auf die Annahme, dass aggressives Verhalten maßgeblich auf Wissensstrukturen, Verhaltensskripte und Schemata zurückgeführt werden kann, die das Ergebnis komplexer sozialer Lernprozesse sind (C. A. Anderson \& Dill, 2000, S. 773). Dabei berücksichtigt das GAM sowohl kurzfristige als auch langfristige Wirkvermutungen.

34 In einer frühen Veröffentlichung wird das Modell noch als General Affective Aggression Model (GAAM) bezeichnet (C. A. Anderson \& Dill, 2000), in späteren Veröffentlichungen nur noch als General Aggression Model (GAM).

35 Für eine detaillierte Darstellung der theoretischen Grundlagen, die für das GAM prägend waren, siehe Anderson und Dill (2000), Kunczik und Zipfel (2004) und Möller (2006). 


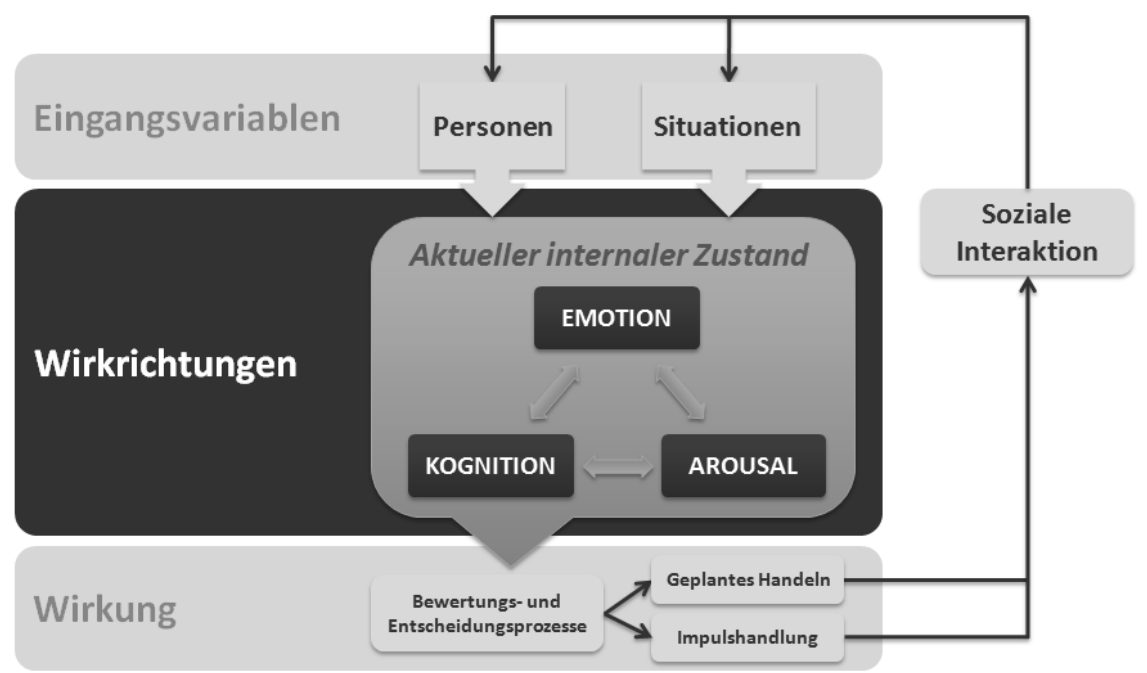

Abbildung 9. Kurzfristige Wirkmechanismen des General Aggression Model (nach C. A. Anderson \& Dill, 2000)

Im Rahmen kurzfristiger Wirkmechanismen (vgl. Abbildung 9) postuliert das GAM, dass situative (z. B. Provokation, Nutzung eines gewalthaltigen Mediums) oder personenbezogene Inputvariablen (z. B. aggressive Persönlichkeit, erhöhte Impulsivität) den aktuellen innerpsychischen Erlebniszustand beeinflussen können. Hinsichtlich dieses Erlebniszustands werden eine kognitive, eine affektive und eine Arousalkomponente unterschieden. So kann es nach dem Modell durch die Nutzung eines gewalthaltigen Computerspiels (situativer Input) zu einer kurzfristigen Aktivierung aggressiver Kognitionen (z. B. Verhaltensskripte), aggressiver Gefühle (z. B. Wut) und der innerphysischen Aktivierungsbereitschaft (Arousal) zu einem aggressionsähnlichen Zustand kommen, der über eine Veränderung von Bewertungs- und Entscheidungsprozessen die Wahrscheinlichkeit für konkret-aggressives Verhalten in der Situation erhöht. Von den innerpsychologischen Erlebniszuständen wird dabei angenommen, dass sie einander wechselseitig beeinflussen: Die Erhöhung einer Komponente (z. B. emotionaler Zustand) erhöht auch die Wahrscheinlichkeit dafür, dass die anderen Komponenten erhöht werden (C. A. Anderson \& Dill, 2000, S. 474).

Die kurzfristigen Wirkmechanismen bedingen nach dem GAM auch langfristige Wirkungen von Mediengewalt (C. A. Anderson \& Dill, 2000; Gentile, et al., 2004). So beschreibt das GAM, dass die wiederholte Nutzung gewalthaltiger Medien zu einer Desensibilisierung für mediale und reale Gewalt sowie über Lernprozesse zu einer Ausdifferenzierung und Verstärkung aggressionsbezogener Wissensstruktu- 
ren führt, indem gewaltlegitimierende Normüberzeugungen, aggressive Wahrnehmungsschemata, feindselige Attributionstendenzen und aggressive Verhaltensskripte gefördert werden (Bushman \& Anderson, 2002, S. 1680). Die Veränderung der kognitiven Wissensstrukturen sowie die Desensibilisierung für Gewalt können in der Folge zur Ausbildung einer aggressiven Persönlichkeitsstruktur und zu einer Reduktion prosozialer Einstellungen beitragen.

Das GAM kann somit als theoretisches Rahmenkonzept veranschaulichen, in welcher Weise gewalthaltige Medien sowohl über kurzfristige als auch langfristige Wirkpfade affektive und kognitive Verarbeitungsprozesse beeinflussen könnten. Diese kurzfristigen und langfristigen Wirkpostulate werden im GAM dafür herangezogen, Aggressivität und schließlich auch die aggressionssteigernde Wirkung von gewalthaltigen Medien zu erklären. Hierbei stellt sich die Frage, ob im GAM nicht auch Zwischenpfade postuliert werden, die neben dem Sozialverhalten auch weitere abhängige Variablen beeinflussen könnten. Buckley und Anderson diskutieren so auch eine Erweiterung des GAM in ein General Learning Model (GLM), indem in ähnlicher Weise wie aggressionsnahe Konstrukte auch andere Inhalte von Computerspielen gelernt werden könnten (vgl. Buckley \& Anderson, 2006). Diese Überlegungen lassen sich jedoch noch weiter fassen: So könnten die kurzfristigen affektiven und kognitiven Veränderungen bei der Nutzung gewalthaltiger Spiele unter bestimmten Voraussetzungen auch einen Einfluss auf Lern- und Informationsverarbeitungsvorgänge nehmen, die außerhalb der Medienrezeptionssituation und ihrer Inhalte stehen. Für die vorliegende Untersuchung ist dabei die kurzfristige erregungssteigernde Wirkung (Arousal) von gewalthaltigen Computerspielen von übergeordneter Bedeutung, da von emotionalen Erlebniszuständen bekannt ist, dass sie kognitive Verarbeitungsprozesse unmittelbar beeinflussen können. Auf die Fragen, durch welche Konstrukte eine solche erregungssteigernde Wirkung gekennzeichnet ist, wie hoch sie ausfällt, wie lang sie bestehen bleibt und durch welche Variablen sie moderiert wird, soll daher im folgenden Kapitel vertiefend eingegangen werden.

Im Anschluss daran soll diskutiert werden, welche emotionalen Erlebniszustände einer erregungssteigernden Wirkung gewalthaltiger Computerspiele zugrunde liegen könnten und warum die Erregung bei gewalthaltigen Computerspielen höher ausfällt als in gewaltneutralen oder gewaltmoderaten Spielen. Hier wird zum einen auf die Wirkannahme des GAM (aggressive Affekte und Kognitionen), zum anderen auf Forschung zu kurzfristigen Angstreaktionen im Zusammenhang mit gewalthaltigen Medien eingegangen. Allerdings wäre eine solche Betrachtungsweise zu einseitig: Starkes Arousal bei Unterhaltungsmediennutzung sollte auch durch positive Erlebniszustände gekennzeichnet sein, da ansonsten nicht erklärt werden könnte, warum sich Personen überhaupt freiwillig gewalthaltigen Computerspielen aussetzen. Um das Bild diesbezüglich zu vervollständigen, soll auch auf theoreti- 
sche Ansätze des Unterhaltungserlebens bei gewalthaltigen Spielen eingegangen werden.

\subsubsection{Allgemeines Erregungspotential (Arousal und Stress)}

"Measurement of arousal as a physiological activation manifesting in different physiological parameters (...) play a special role in estimating the effects of video games on people's mental processes and behavior" (Staude-Müller, Bliesener, \& Luthman, 2008, S. 42).

Einen Schwerpunkt in der empirischen Medienwirkungsforschung zu emotionalen Reaktionen auf gewalthaltige Medien bilden Studien, die als abhängige Variable Arousal zum Gegenstand haben. Unter Arousal wird eine vom Ruhewert des Individuums abweichende, physiologische, psychophysiologische und/oder psychische Erregung bzw. Aktivierungsbereitschaft verstanden (Fröhlich, 2000, S. 164). Arousalparameter werden häufig eingesetzt, um allgemeine Erregungsverläufe im Rahmen der Medienrezeption abzubilden. In der (De)Sensibilisierungsforschung werden dem Arousal vergleichbare Konstrukte häufig verwendet, um Erregungen im Anschluss an kurzfristige oder langfristige Expositionsereignisse von Mediengewalt zu untersuchen und hieraus Rückschlüsse auf Veränderungen in der psychophysiologischen Aktivierungsbereitschaft zu ermöglichen (vgl. Bartholow, Bushman, \& Sestir, 2006; Carnagey, Anderson, \& Bushman, 2007; Staude-Müller, et al., 2008). Die Erfassung von Arousal erfolgt in der Regel über biopsychologische Erlebniskorrelate, am häufigsten über die kardiovaskulären Parameter Herzfrequenz und Blutdruck, aber auch über Hautwiderstandsmessung, Atemfrequenz, Körpertemperatur, hormonelle Untersuchungen des Adrenalin-, Noradrenalin, Cortisol oder Testosteronspiegels oder Selbstberichtsinstrumente. Die meisten Untersuchungen sind dem Forschungsgegenstand entsprechend experimentelle oder quasiexperimentelle Laboruntersuchungen.

Erste Studien zur Arousal induzierenden Wirkung gewalthaltiger Spiele wurden bereits in den 1980er Jahren durchgeführt (für einen Überblick vgl. Ballard \& Wiest, 1996, S. 719). Eine Metaanalyse von Anderson und Bushman (2001) gelangte auf Basis von sieben bis zum Publikationsjahr 2000 vorliegenden experimentellen Untersuchungen zu dem Ergebnis, dass die durch das GAM postulierte Annahme einer Erhöhung physiologischen Arousals durch gewalthaltige Spiele von der empirischen Forschung insgesamt gestützt wird $(r=.22)$. Dies traf auf die kardiovaskulären Arousalparameter Herzschlag und Blutdruck in gleicher Weise zu (C. A. Anderson \& Bushman, 2001, S. 358). Eine Aktualisierung dieser Metaanalyse durch Anderson (2004) ermittelte zu neun bis zum Publikationsjahr 2004 vorliegenden Untersuchungen eine etwas geringere Korrelation zwischen Gewalt- 
spielnutzung und Arousal $(r=.18)$. Mittels eines Kriterienkatalogs konnte in dieser Studie nach methodisch hochwertigen und methodisch problematischen Studien differenziert werden. ${ }^{36}$ Dabei zeigte sich für Studien, die alle definierten Qualitätskriterien erfüllen, ein höherer positiver Zusammenhang zwischen Gewaltspielnutzung und Arousal ( $r=.22)$ als bei methodisch problematischen Studien $(r=.10)$, die durch mindestens einen methodischen Mangel gekennzeichnet waren (C. A. Anderson, 2004).

Damit deuten beide Metaanalysen insgesamt auf einen positiven Zusammenhang zwischen Gewaltspielnutzung und Arousal hin. Nicht ersichtlich wird aus diesen Übersichtsarbeiten jedoch, inwieweit die erregungssteigernde Wirkung gewalthaltiger Medien auch ein relevantes Stressgeschehen anzeigen könnte. Moderatoranalysen wurden im Rahmen der Metaanalysen aufgrund der geringen Studienzahl ebenfalls nicht durchgeführt (C. A. Anderson \& Bushman, 2001, S. 358). Es wurde deshalb entschieden eine ergänzende Sichtung des Forschungsstandes zur Nutzung gewalthaltiger Computerspiele und verschiedener Arousalparameter vorzunehmen. ${ }^{37} \mathrm{Um}$ hier auch Aussagen zur erregungssteigernden Wirkung von gewalthaltigen im Gegensatz zu gewaltneutralen Spielen zuzulassen, wurden nur solche Studien betrachtet, die als Kontrollbedingungen keine Ruhebedingungen, sondern gewaltneutrale oder gewaltreduzierte Spielbedingungen realisiert haben. ${ }^{38}$

Hinsichtlich kardiovaskulärer Arousalparameter konnten neun Untersuchungen aufgefunden werden, die gewaltneutrale oder gewaltreduzierte Kontrollbedingungen vorgesehen haben. In acht Untersuchungen ergab sich ein erhöhtes Wirkpotential gewalthaltiger gegenüber gewaltneutraler Spiele (Baldaro, et al., 2004; Ballard \& Wiest, 1996; Barlett, Branch, Rodeheffer, \& Harris, 2009; Barlett \& Rodeheffer, 2009; Brady \& Matthews, 2006; Fleming \& Rickwood, 2001; Frindte \& Obwexer, 2003; Maass, Lohaus, \& Wolf, 2010). Nur in einer Studie konnten hierzu abweichende Resultate aufgefunden werden, indem sich in allen Spielbe-

36 Methodische Mängel wurden beispielsweise dann angenommen, wenn die Gewaltbedingung nur wenig Gewalt beinhaltete oder Unterschiede im Arousal zwischen Gewalt- und gewaltneutraler Bedingung zwar erfasst wurden, vorab aber ein Angleich des Erregungslevels zwischen den Bedingungen (z. B. durch Pretesting und Stimulusauswahl) angestrebt wurde (vgl. C. A. Anderson, 2004, S. 116).

37 Die Recherche wurde über die Datenbanken PubMed, PsyCONTENT und Google Schoolar durchgeführt. Es wurden deutsche und englische Suchbegriffe eingesetzt. Dabei wurde die UV-Ebene computerspiel, videospiel, computer game, video game mit den Begriffen arousal, physiology, cortisol, stress, erregung der AV-Ebene kombiniert. Zusätzlich wurden im "Schneeballverfahren" Studien anhand vorhandener Quellennachweise einbezogen.

38 So sollte eine Erregungserhöhung im Vergleich zu Ruhebedingungen bei nahezu jeder Form von Aktivität zu beobachten sein, so auch bei der Nutzung von Computerspielen, ob gewalthaltig oder nicht. In einer aktuelleren Untersuchung konnte so z. B. gezeigt werden, dass bei der Nutzung eines gewaltneutralen Rennspiel der diastolische und systolische Blutdruck über die Treatmentdauer von 24 Minuten mehr als zwei Standardabweichungen gegenüber einer Ruhebedingung erhöht waren (vgl. Borusiak, Bouikidis, Liersch, \& Russell, 2008). 
dingungen ein Abfall kardiovaskulärer Arousalparameter gegenüber einer Prämessung zeigte (Staude-Müller, et al., 2008).

In vier der aufgefundenen Untersuchungen wurden die Wirkungen eines gewalthaltigen unmittelbar mit denen eines gewaltneutralen Spiels verglichen. Zwei dieser Studien stellen Within-Subject-Designs mit kindlichen (Fleming \& Rickwood, 2001) und erwachsenen Probanden (Frindte \& Obwexer, 2003) dar und zwei Studien Between-Subject-Designs mit erwachsenen Probanden (Baldaro, et al., 2004; Barlett, Branch, et al., 2009). Alle vier Studien bestätigen ein erhöhtes Arousal der eingesetzten Gewaltspielbedingung gegenüber der Gewaltneutralbedingung, wobei sich bei Baldaro und Kollegen (2004) dieser Unterschied nur hinsichtlich des Blutdrucks, nicht aber der Herzfrequenz zeigte. In weiteren vier Untersuchungen wurden gewalthaltige Spiele nicht mit gewaltneutralen sondern mit gewaltreduzierten Spielen verglichen. Brady und Matthews (Brady \& Matthews, 2006) ermittelten anhand einer Stichprobe von N = 100 männlichen Studenten eine Erhöhung des diastolischen und des systolischen Blutdrucks in einer 10-minütigen Gewaltspielbedingung (Grand Theft Auto III) gegenüber einer Spielbedingung mit moderater Gewalt (The Simpsons: Hit and Run) und dies auch unter Kontrolle einer Vielzahl von Kovariaten. Es zeigte sich ein Anstieg des systolischen Blutdrucks in der Gewaltbedingung, der jedoch nur auf jene Männer zutraf, die in ihrem Umfeld einem hohen Maß an Gewalt ausgesetzt waren (Brady \& Matthews, 2006). Barlett und Rodeheffer (2009) gelangten zu dem Befund, dass die Herzfrequenz beim Spielen eines Spiels mit realistischer Gewalt stärker ausfiel als bei einem Spiel mit unrealistischer (Fantasy)Gewalt und dass diese Erregungserhöhung nicht nur initial, sondern während des gesamten 45-minütigen Treatments zu beobachten war. Ballard und Wiest (1996) nutzten ein gewaltneutrales Billardspiel sowie eine gewaltreduzierte und eine besonders gewalthaltige Variante des Prügelspiel Mortal Kombat. Die beiden gewalthaltigen Spiele unterschieden sich dabei nur dahingehend, dass die besonders gewalthaltige Variante Blut und Splattereffekte als Gewaltfolgen darstellte. Damit wurde in dieser Studie die Gewalt systematisch innerhalb eines ansonsten einheitlichen Unterhaltungsangebots manipuliert. An $\mathrm{N}=30$ Studenten wurde die differenzielle Wirkung der drei Computerspiele auf Blutdruck und Herzfrequenz untersucht. Ein erhöhter systolischer Blutdruck ergab sich nur für Probanden, die die besonders gewalthaltige Mortal-Kombat-Version mit Blut und Splattereffekten spielten. Spieler der milderen Variante von Mortal Kombat wiesen hingegen keinen erhöhten Blutdruck gegenüber den Billardspielern auf. Spieler beider Mortal-Kombat-Versionen wiesen jedoch einen erhöhten Herzschlag gegenüber den Billardspielern auf und unterschieden sich dabei nicht signifikant voneinander (Ballard \& Wiest, 1996). In der Studie von Staude-Müller und Kollegen (2008) ergab sich, abweichend von den anderen Studien, ein Abfall von Herz- und Atemfrequenz beim Spielen eines hinsichtlich seines Gewaltgehal- 
tes variierten Computerspiels (Unreal Tournament 2003) gegenüber der Baselinemessung vor dem Spielen. Dieser Befund könnte jedoch dadurch zu erklären sein, dass während der Baselinemessung zeitgleich die Instruktion und Befragung der Probanden stattfand, und sich dadurch bereits ein über das Ruhelevel hinausgehendes Arousal eingestellt haben könnte. Deskriptiv ist den Daten zu entnehmen, dass das Arousal besonders stark in der gewaltreduzierten Treatmentbedingung abfällt (Staude-Müller, et al., 2008).

In zwei weiteren Untersuchungen wurde nicht nur das Arousal in verschiedenen Spielbedingungen miteinander verglichen, sondern ereigniskorrelierte Arousalwirkungen verschiedener Spielereignisse innerhalb der Spielbedingungen analysiert (Ravaja, Turpeinen, Saari, Puttonen, \& Keltikangas-Jarvinen, 2008; Salminen \& Ravaja, 2008). Damit können diese Untersuchungen zusätzliche Hinweise darauf geben, ob in gewalthaltigen Spielen auch tatsächlich die Gewaltereignisse selbst von Bedeutung für erhöhte Arousalreaktionen sind und nicht etwa andere Elemente wie zum Beispiel erhöhte Action und Spielgeschwindigkeit. Ravaja, Saari, Laarni, Kallinen, und Salminen (2005) untersuchten die Arousal- sowie emotionale Reaktionen von $\mathrm{N}=36$ Studenten auf Erfolgs- und Misserfolgsereignisse in einem gewaltneutralen und einem gewalthaltigen Computerspiel. Dabei zeigte sich, dass das Arousal ereigniskorreliert mit Gewalthandlungen besonders stark ausfiel. Mittels einer Facial Electromyography (EMG) wurde zusätzlich ermittelt, ob die Arousalreaktionen von positiv oder negativ valenten Emotionen begleitet waren. Während Spielerfolge im gewaltneutralen Spiel (Aufsammeln von Bananen) wie erwartet mit valent positiven Emotionen einhergingen waren Spielerfolge im gewalthaltigen Spiel (Töten oder Verwunden des Gegners) mit negativen Emotionen verbunden. Der Tod der eigenen Spielfigur war im gewalthaltigen Spiel hingegen mit positiven Emotionen verbunden (Ravaja, et al., 2005). Wie in einer späteren Veröffentlichung der Arbeitsgruppe gezeigt wurde, traten positive Reaktionen jedoch auch bei Verlust der eigenen Spielfigur im gewaltneutralen Spiel auf(Ravaja, et al., 2008). Die Autoren deuten die Befunde als Hinweis auf eine physiologische Entspannungsreaktion nach Beendigung eines kontinuierlichen Stressors. Im Experiment ebenfalls verwendete EEG-Messungen $(\mathrm{N}=25)$ konnten die erhöhte Arousalreaktion während der Gewaltereignisse im eingesetzten First-Person-Shooter ebenfalls dokumentieren (Salminen \& Ravaja, 2008).

Die Ergebnisse zur Arousalwirkung gewalthaltiger Computerspiele werden durch einige Studien zur rezeptionsbedingten Ausschüttung von Stresshormonen (Adrenalin, Nor-Adrenalin, Cortisol) ergänzt. Diese konnten insbesondere Reaktionen innerhalb der katecholaminen Stressachse nachweisen (vgl. Kapitel 1.4.2). Skosnik und Kollegen (2000) untersuchten die Auswirkungen einer 15-minüten Gewaltspielrezeption auf erwachsene Probanden und kontrollierten hierbei die Stressindikatoren Nor-Adrenalin und Cortisol. Die zur Messung beider Parameter 
erforderlichen Speichelproben wurden vor dem Experiment, unmittelbar nach der Computerspielphase sowie 20 Minuten später im Anschluss an das Treatment entnommen. Es ergab sich ein Anstieg des Noradrenalinspiegels, jedoch kein Anstieg des Cortisolspiegels, was die Autoren in der Weise interpretieren, dass die Computerspielgewalt möglicherweise zu mild oder aber die Verzögerung der Cortisolausschüttung zu groß ist, um bereits 20 Minuten nach dem Stressor erfasst werden zu können. Auch in einer anderen Untersuchung an Kindern zeigte sich nur eine Erhöhung kardiovaskulärer Parameter, nicht aber der Cortisolwerte, obgleich hierbei ein extrem gewalthaltiges Spiel zum Einsatz kam (Ivarsson, Anderson, Åkerstedt, \& Lindblad, 2009 a, 2009 b). Eine besonders umfassende Untersuchung zur Wirkung gewalthaltiger Medien auf kardiovaskuläre und hormonelle Stressparameter berichten Maass, Lohaus und Wolf (2010). In dieser Studie wurden als Stimulusmaterial gewaltneutrale und gewalthaltige Filme und Computerspiele eingesetzt und damit ein $2 \times 2$ Design realisiert. Abgesehen vom Cortisol deuten alle erhobenen Parameter auf ein erhöhtes Stresserleben im Rahmen der Gewaltrezeption hin. Die Cortisolwerte sanken stattdessen in allen Bedingungen gegenüber der Prämessung ab, wenngleich interindividuelle Variabilität in diesen Daten besteht, und einige Jungen mit erhöhten Cortisolreaktionen auf die Medienbedingungen reagierten (Maass, et al., 2010, S. 17 ff.). Post Hoc zeigt sich eine erhöhte Cortisolausschüttung in der gewalthaltigen Filmbedingung gegenüber der gewaltneutralen Spielbedingung sowie insgesamt stärkere Cortisolreaktionen in den Gewalt- gegenüber den gewaltneutralen Bedingungen. Eine andere Studie konnte hingegen Cortisolreaktionen in Folge der Nutzung eines gewalthaltigen Computerspiels nachweisen, wenngleich in dieser nicht zwischen gewalthaltigen und gewaltneutralen Spielen differenziert wurde sondern stattdessen die akustische Präsentation eines gewalthaltigen Spiels systematisch variiert wurde (Hébert, Béland, DionneFournelle, Crête, \& Lupien, 2005). Hier zeigten sich erhöhte Cortisolreaktionen erwachsener Probanden, die ein First-Person-Shooterspiel mit Musikeffekten spielten, gegenüber den Probanden, die das selbe Spiel ohne Musikeffekte nutzten.

Die Befunde zum Zusammenhang von Gewaltcomputerspielnutzung und Arousal stehen insgesamt in Übereinstimmung mit dem Kenntnisstand zu gewalthaltigen Filmen. So konnte etwa gezeigt werden, dass gewalthaltige Filme zu stärkerem Arousal führen als gewaltneutrale (vgl. Bushman \& Geen, 1990; Murray, et al., 2006) und dass Arousalreaktionen unmittelbar mit Gewaltereignissen im Film korrelieren (vgl. J. Grimm, 1996; Mangold, Winterhoff-Spurk, Stoll, \& Hamann, 1998). Es gibt jedoch einige Gründe für die Vermutung, dass Arousalreaktionen bei Computerspielgewalt übergreifend stärker ausfallen könnten als bei Filmgewalt. Hier ist zunächst die aktivere Beteiligung sowie erhöhte kognitive Beanspruchung des Rezipienten beim Computerspielen anzuführen. Gerade in Actionspielen ist der Spieler häufig dem Druck ausgesetzt, möglichst schnell zu handeln und 
Aufgaben unter Zeitdruck und der Bedrohung oder Konkurrenz anderer Spielfiguren und Mitspieler zu bewältigen (vgl. Kapitel 1.3.1). Hinzu kommt die hohe Ereignisfrequenz von Gewaltereignissen in gewalthaltigen Computerspielen im Vergleich zu Filmen (vgl. Kapitel 1.1.2). Von den aufgefundenen Untersuchungen erlaubt nur eine einzige Aussagen über die differenzielle Erregungswirkung von gewalthaltigen Spiel- und Filmbedingungen (Maass, et al., 2010). Diese gelangt zu gemischten Befunden, indem Computerspiel- und Filmgewalt in gleichem Maße zu einer erhöhten Adrenalinausschüttung führten, hinsichtlich der Herzfrequenz und der Herzfrequenzvariabilität jedoch eine besonders hohe Erregungsreaktion in der gewalthaltigen Spielbedingung anzeigten. Die höchste Cortisolreaktion ergab sich hingegen in der gewalthaltigen Filmbedingung (Maass, et al., 2010).

Wenngleich damit der Forschung eine überwiegende Einigkeit hinsichtlich einer erhöhten Erregungswirkung gewalthaltiger gegenüber gewaltneutraler Computerspiele entnommen werden kann, bleiben noch wichtige Fragen offen. Zum einen ist unklar, wie die Stärke der Erregungswirkung einzuschätzen ist und damit auch, ob die physiologischen Daten ein relevantes Stresserleben anzeigen. Dies ist anhand der zur Verfügung stehenden Daten noch nicht eindeutig zu beurteilen, da die Studien zu kardiovaskulären Arousalparametern nur grobe Indikatoren bereitstellen, die hinsichtlich ihrer Ausprägungsintensität nicht eindeutig zu interpretieren sind. Insgesamt lässt sich jedoch feststellen, dass bereits eine 10-20 minütige Nutzung eines gewalthaltigen Computerspiels zu einem messbaren Anstieg des physiologischen Arousals führt (vgl. Barlett, Branch, et al., 2009, S. 234). Zudem deuten insbesondere die wenigen vorhandenen Studien zu stresshormonellen Parametern darauf hin, dass in Folge der Nutzung gewalthaltiger Spiele Adrenalin und Noradrenalin ausgeschüttet und damit die katecholamine Stressachse aktiviert wird (Maass, et al., 2010; Skosnik, et al., 2000). Ein Nachweis von Cortisolreaktionen in Folge der Nutzung gewalthaltiger Spiele ist bislang jedoch, mit Ausnahme der Studie von Hébert und Kollegen (2005), ausgeblieben. Gerade beim Cortisol sollte jedoch bedacht werden, dass auch von anderen relevanten und inzwischen gut erforschten relativen Stressoren (vgl. Kapitel 1.4.2) bekannt ist, dass im Labor nicht immer der Nachweis einer Cortisolreaktion erbracht werden kann, sondern Personen auf diese sehr heterogen reagieren und eine zuverlässige Erfassung zudem eine konsequente Berücksichtigung circadiane Periodizitäten der Cortisolausschüttung voraussetzt. Unter den drei aufgefundenen Studien, die Cortisolreaktionen in Folge der Rezeption gewalthaltiger Spiele untersucht haben, lassen zwei Studien zudem Zweifel hinsichtlich des Gewaltgrades der eingesetzten Angebote aufkommen. So wird in der Studie von Skosnik und Kollegen (2000) nicht dokumentiert, welches Spiel eingesetzt wurde, sondern hier nur darauf hingewiesen, dass es sich um ein "shooterähnliches" Spiel handelte, in dem Gewalt gegen Menschen eingesetzt wird (vgl. Skosnik, et al., 2000, S. 62). Angesichts der großen Bandbreite 
möglicher Realitäts- und Visualisierungsgrade erscheint diese Angabe wenig aussagekräftig. In der Studie von Maass und Kollegen (2010) wurde mit King Kong ein Spiel mit einer Altersfreigabe ab 12 Jahren eingesetzt, das für die untersuchte Population von Kindern zwar als forschungsethisch angemessen, jedoch allenfalls als gewaltmoderat gelten kann. Somit verbleibt mit der Studie von Ivarsson und Kollegen (Ivarsson, et al., 2009 a, 2009 b) nur eine einzige Studie, bei der die Auswirkungen von extremer Computerspielgewalt auf den Cortisolspiegel untersucht wurden. Angesichts der geringen empirischen Datenbasis sowie der gegenläufigen Befunde zum Arousal und zu katecholaminen Stressreaktionen erscheinen in jedem Fall weitere Studien notwendig, um zuverlässig einschätzen zu können, ob gewalthaltige Computerspiele als relevante Stressoren wirksam werden können. Legt man die Annahme zugrunde, dass sich verschiedene Personen dahingehend unterscheiden könnten, in welcher Weise und wie stark sie auf mediale Gewalt reagieren, erscheint es berechtigt anzunehmen, dass interindividuell sowohl moderate Arousalreaktionen als auch intensive Stressreaktionen möglich sind. Diese Überlegungen sollen in den nachfolgenden Kapiteln zu spezifischen emotionalen Wirkungen gewalthaltiger Spiele sowie im Kapitel 1.4.4 zu moderierenden Faktoren des Erregungspotentials gewalthaltiger Computerspiele erneut aufgegriffen werden.

Auch der zeitliche Verlauf der Erregungsreaktion während und nach der Rezeption ist anhand der Datenlage schwer einzuschätzen. Zwar zeigte sich bei Frindte und Obwexer (2003), dass die Erregung nach der gewaltneutralen Spielbedingung unter das Baselineniveau abfiel, während die Erregung nach dem gewalthaltigen Spiel weiterhin über dem Baselineniveau verblieb. Aus der Studie wird jedoch nicht ersichtlich, in welchem zeitlichen Abstand nach dem Treatment diese Abschlussmessung stattgefunden hat. In der Studie von Barlett und Rodeheffer (2009), in der insgesamt vier Messzeitpunkte zur Herzfrequenz realisiert wurden, zeigte sich nach einem initialen Anstieg in der realistischen Gewaltbedingung eine Stabilisierung der erhöhten Erregung über die Zeitdauer der Rezeption. In einer weiteren Untersuchung konnten Barlett und Kollegen (2009) zeigen, dass eine durch ein gewalthaltiges Computerspiel erhöhte Herzfrequenz noch vier bis neun Minuten nach Beendigung des Treatments anhielt. Beide Studien berücksichtigten jedoch nur Herzfrequenzmessungen und erlauben aufgrund des Verzichts auf breitbandige Messansätze keine Aussagen über die Stabilität anderer Arousalparameter. Ergänzende Hinweise lassen sich Studien entnehmen, die das Schlafverhalten in der Folgenacht an die Nutzung gewalthaltiger Computerspiele zum Gegenstand bzw. mit erhoben haben. Hier wurden erhöhte kardiovaskuläre Aktivitäten (Higuchi, Motohashi, Liu, \& Maeda, 2005; Ivarsson, et al., 2009 a, 2009 b) und eine Reduzierung von Slow-Wave Sleep Patterns (Dworak, et al., 2007) und REMSchlafphasen (Higuchi, et al., 2005) beobachtet. In einer weiteren Studie konnte gezeigt werden, dass in Folge langanhaltenderer Spielphasen (7 Stunden über 3 Ta- 
ge) eines einfachen Denkspiels (Tetris) während der nachfolgenden Einschlafphasen hypnagoge Bilder des Spiels wiedererlebt wurden (Stickgold, Malia, Maguire, Roddenberry, \& O'Connor, 2000). Dieser Befund fand sich unabhängig von der Spielerfahrung mit Tetris und sowohl bei gesunden Patienten als auch bei Amnestikern (Stickgold, et al., 2000). Wenngleich eine systematische Einordnung und Bewertung dieser sehr differenziellen und zum Teil auch exotisch anmutenden Befunde aufgrund der geringen Zahl von Untersuchungen kaum möglich erscheint, könnten diese Daten zumindest als Hinweise dafür verstanden werden, dass die affektive Verarbeitung des Computerspielens und damit in Zusammenhang stehende Erregungsverläufe deutlich über die eigentliche Rezeptionssituation hinauswirken könnten. 


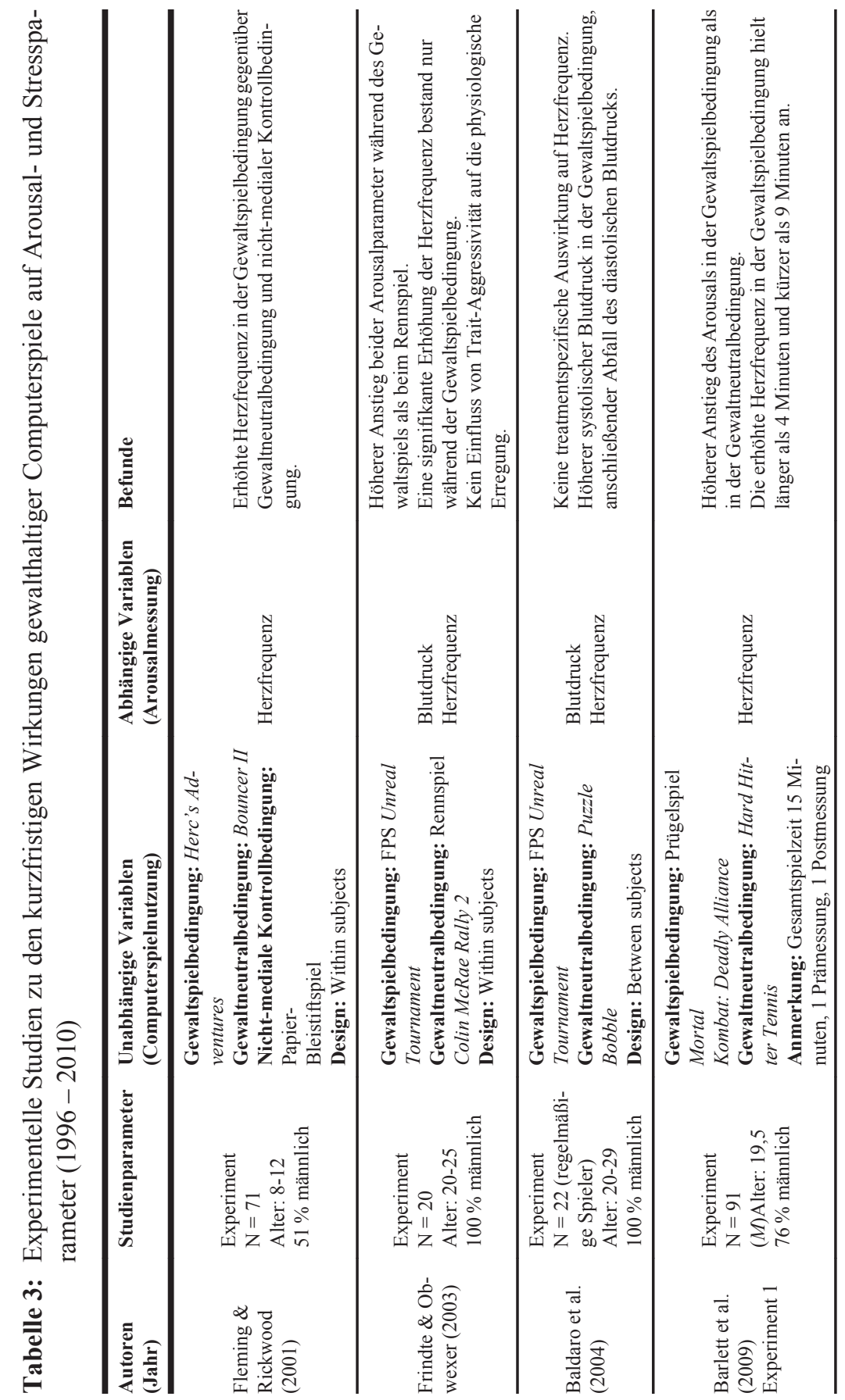




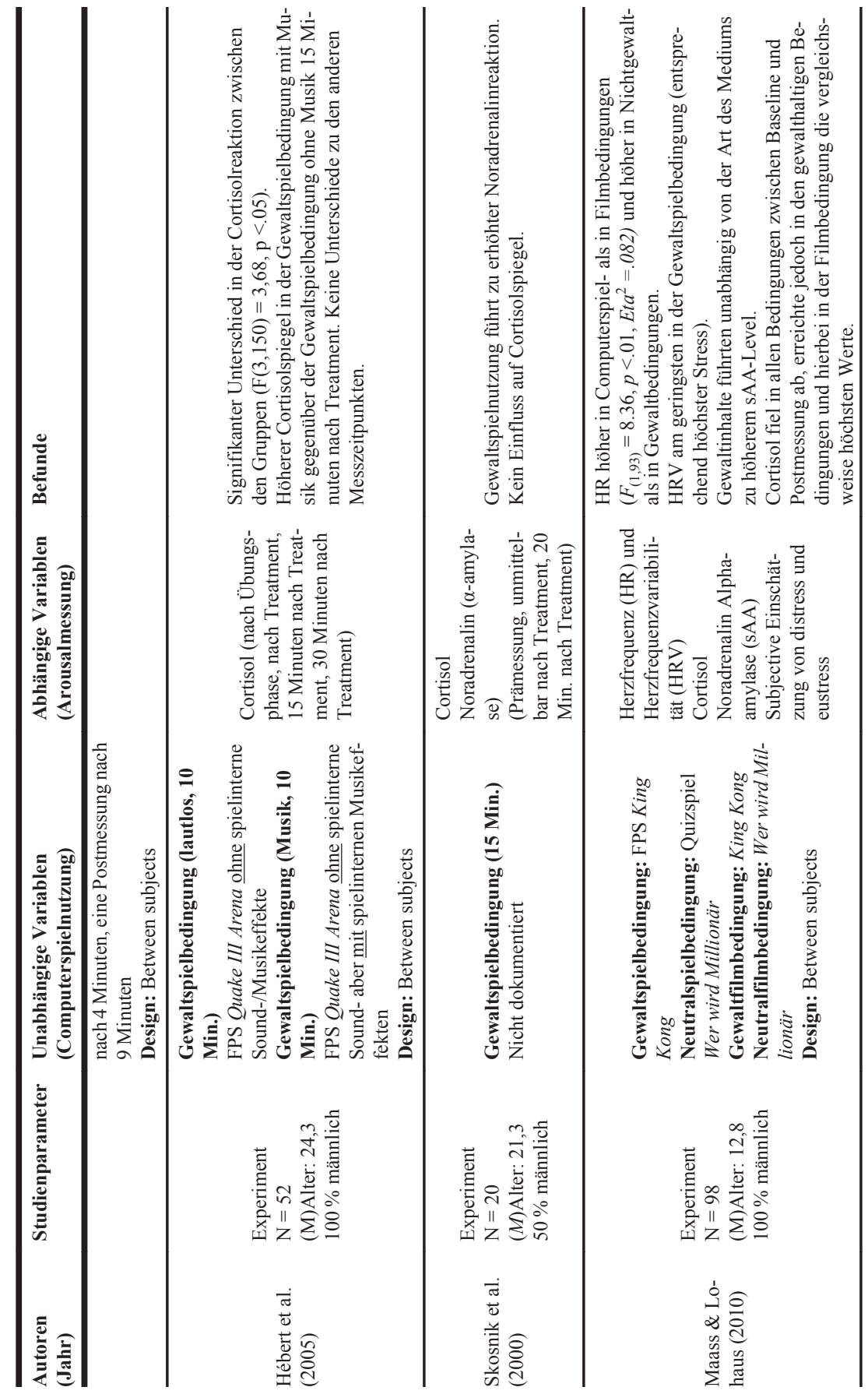




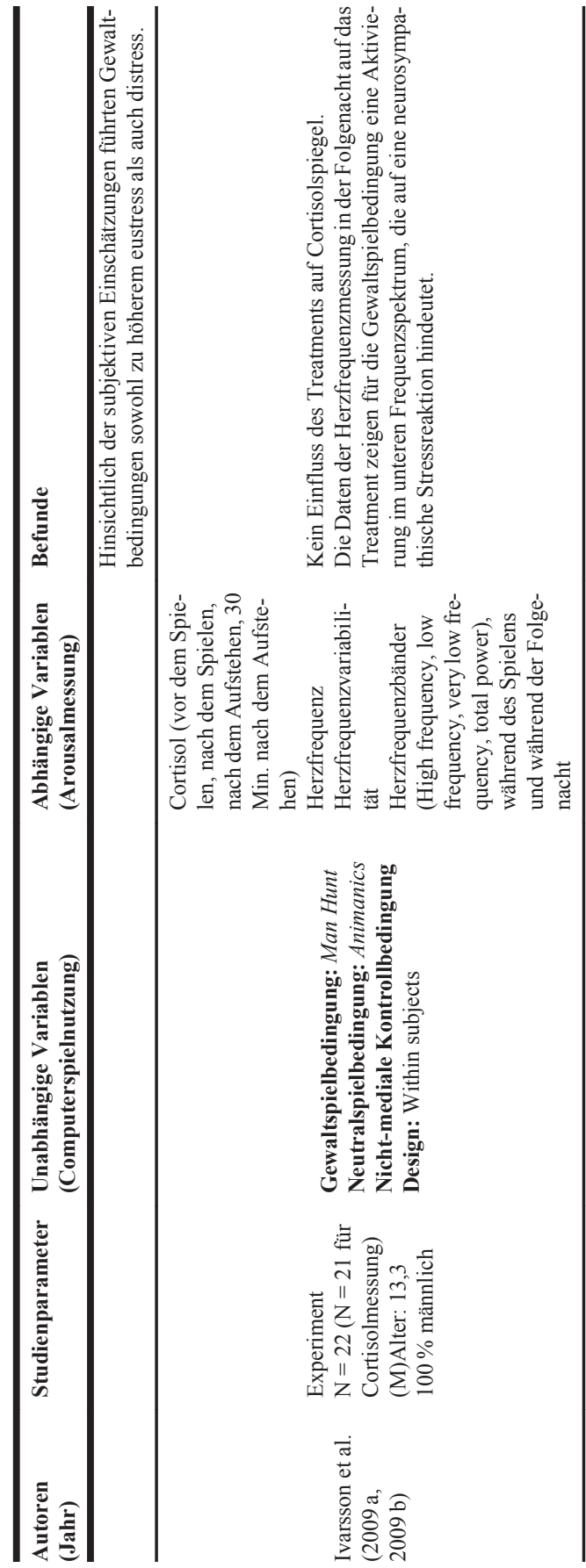

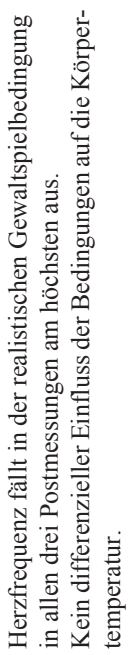

离苛站

品

范 总

总 曹

๑)

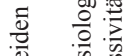

$\infty$ \&

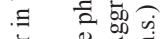

芩

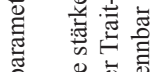

空 on

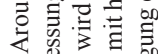

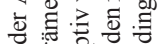
跑语

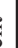

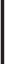

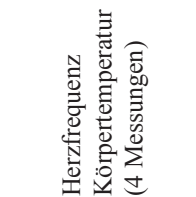

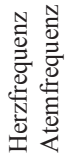

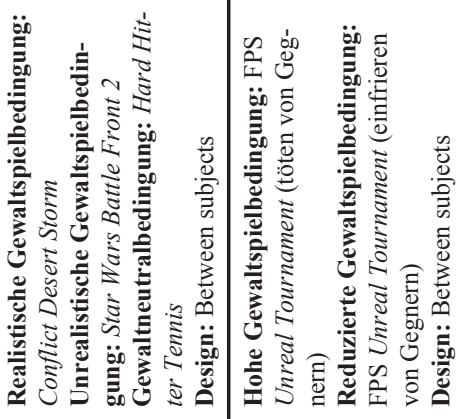

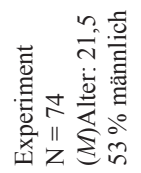

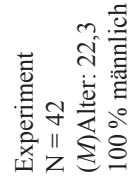

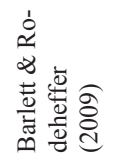

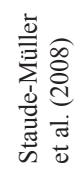




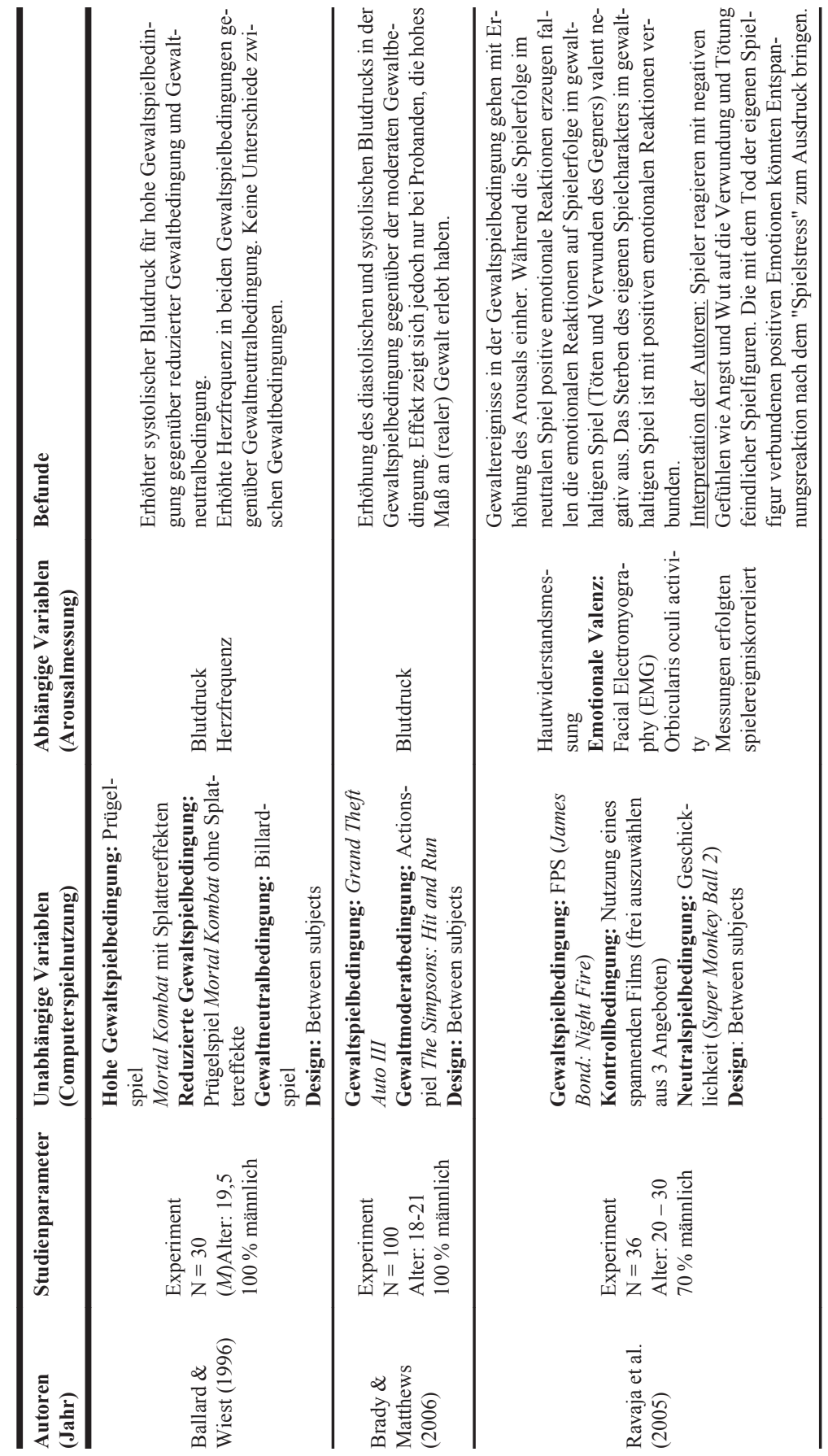




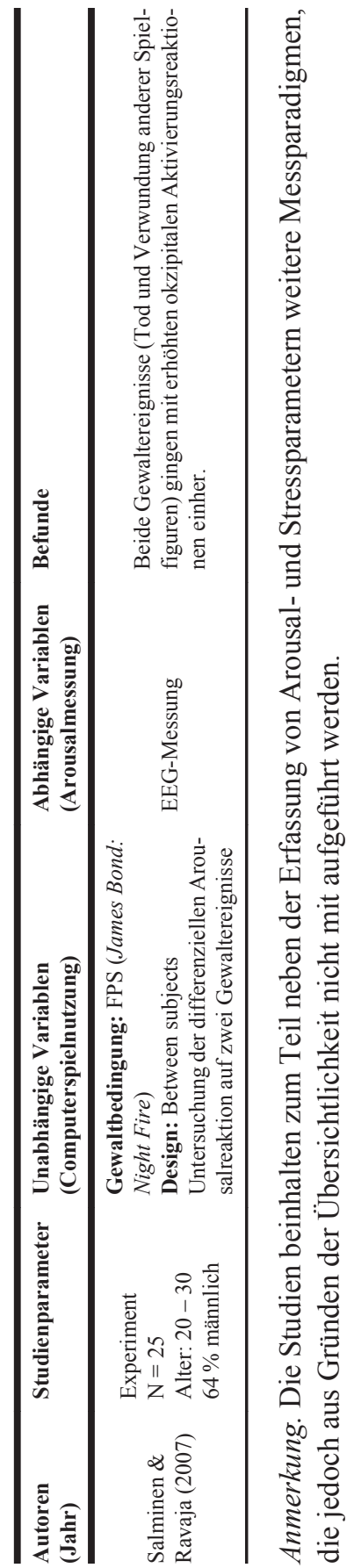




\subsection{State-Aggressivität}

Dem GAM zufolge können gewalthaltige Medien aggressive Kognitionen und aggressive Affekte kurzfristig verstärken (C. A. Anderson \& Bushman, 2001; C. A. Anderson, 2004; Bushman \& Anderson, 2002). Dabei wird angenommen, dass sich die kurzfristigen Wirkungen wechselseitig verstärken, indem Arousal als "aggressionsähnlicher Zustand" die Wahrscheinlichkeit für aggressive Gedanken und Gefühle erhöht und diese wiederum ein höheres Arousal befördern (vgl. Buckley \& Anderson, 2006, S. 372). Die Annahme kurzfristiger Verstärkung aggressiver Kognitionen stützt sich maßgeblich auf Erkenntnisse zum impliziten Lernen und hierbei insbesondere dem Priming. So wird angenommen, dass durch aggressionsbezogene Reize in Computerspielen (z. B. Darstellungen von Waffen, Kampfszenarien und Gewalthandlungen) aggressionsbezogene Gedanken stimuliert werden, die in der Folge die Zugänglichkeit weiterer aggressiver Gedanken im Sinne eines processing bias ${ }^{39}$ wahrscheinlicher machen (vgl. Kirsh \& Mounts, 2007, S. 354). Wie das Arousal wurden auch diese kognitiven Wirkannahmen nahezu ausschließlich im Rahmen experimenteller Studien untersucht.

Aggressive Kognitionen werden mittels vielfältiger Methoden erfasst (vgl. Kunczik \& Zipfel, 2004, S. 203), die sich insgesamt jedoch zwei grundsätzlichen Zugangswegen zuordnen lassen. Die erste Operationalisierungsform stellen WortVervollständigungs-Tests dar (C. A. Anderson, et al., 2004; Barlett \& Rodeheffer, 2009). Hier werden die Probanden dazu aufgefordert fehlende Buchstaben unvollständiger Wörter zu ergänzen, wobei jeweils eine vergleichbare Anzahl gewaltneutraler Wörter als auch gewaltbezogener Worte möglich ist (Beispiele: "K I _ _" wird zu "KISS" oder "KILL", "E X P L O _ E" wird zu "EXPLORE" oder "EXPLODE"). Hiermit soll überprüft werden, inwieweit gewaltbezogene Kognitionen aufgrund eines vorangegangenen Primings situativ in besonderer Weise verfügbar bzw. zugänglich sind. Eine ähnliche Intention liegt Reaktionstests zu aggressionsbezogenen Wörtern zugrunde, in denen Probanden dazu aufgefordert werden eingeblendete Wörter so schnell wie möglich vorzulesen (vgl. C. A. Anderson \& Dill, 2000). Dabei werden aggressionsbezogene Wörter (z. B. Mörder) und nicht-aggressionsbezogene Kontrollwörter eingeblendet. Die Differenz in der Geschwindigkeit des Vorlesens aggressionsbezogener und Kontrollwörter wird als Maß für die aktuelle Verfügbarkeit aggressiver Kognitionen herangezogen (C. A. Anderson

39 Diese veränderte Ausrichtung der Informationsverarbeitung kann so erklärt werden, dass durch das vorangegangene Priming aggressionsbezogener Informationen diesem "Wissensbereich" stärkere Aufmerksamkeitsressourcen zugeordnet werden, die zu einer bevorzugten Verarbeitung dieser Informationen und in diesem Sinne zu einem processing bias führen (Kirsh \& Mounts, 2007, S. 354). 
\& Dill, 2000, S. 784). Eine weitere Operationalisierungsform aggressiver Kognitionen stellt die Messung feindseliger Attributionstendenzen (hostile attribution bias) dar. Hier werden in der Regel Vignettentechniken eingesetzt, bei denen $\mathrm{Zu}$ schreibungen zu anderen Personen untersucht werden können (Bushman \& Anderson, 2002; Kirsh, 1998). Kirsh (1998) setzte im Rahmen eines Experimentes mit kindlichen Versuchsteilnehmern kurze Geschichten ein, die unterschiedliche provokative Handlungen gleichgeschlechtlicher Personen in ähnlichem Lebensalter beinhalteten (z. B. Kind wird mit einem Ball am Rücken getroffen). Anschließend wurden zwei Fragen zu der Intention des Schädigers, zwei Fragen zu geeigneten Reaktionen und Bestrafungen und zwei Fragen zur emotionalen Verfassung des Schädigers gestellt. In ähnlicher Weise gingen Bushman und Anderson (2002) im Rahmen eines Experimentes mit jugendlichen Versuchsteilnehmern vor. Hier lasen die Probanden kurze Geschichten, in denen sich die jeweils handelnde Person mit einem bestimmten Problem konfrontiert sah (z. B. Autounfall mit Blechschaden). Anschließend wurden die Probanden aufgefordert 20 Dinge anzugeben, die die handelnde Person als nächstes tun, sagen, denken oder fühlen würde (Bushman \& Anderson, 2002). Die Antworten auf die Vignetten werden in der Regel inhaltsanalytisch ausgewertet (Bushman \& Anderson, 2002; Kirsh, 1998).

Zur Messung aggressiver Affekte werden häufig Ärger-Zustands-Skalen zur Selbstbeurteilung des aktuellen Ärgeraffektes eingesetzt (C. A. Anderson \& Dill, 2000; Ballard \& Wiest, 1996; Fleming \& Rickwood, 2001; Frindte \& Obwexer, 2003; Panee \& Ballard, 2002). Eine häufig eingesetzte Skala ist zum Beispiel die situative Ärgerkomponente des State-Trait-Ärgerausdrucks-Inventars (Schwenkmezger, Hodapp, \& Spielberger, 1992).

Die Wirkungen gewalthaltiger Computerspiele auf aggressive Kognitionen und Affekte wurden im Rahmen vielfältiger Studien untersucht, die in zwei Metaanalysen zusammengefasst wurden. Anderson und Bushman bereiteten die Forschungsdaten zu 20 Studien auf, die aggressive Kognitionen und 17 Studien, die aggressive Affekte in Folge der Nutzung gewalthaltiger Spiele untersuchten. Hinsichtlich beider Konstrukte ergaben sich positive Korrelationen mit Gewaltspielnutzung (vgl. Tabelle 4). 
Tabelle 4. Befunde der Metaanalyse von Anderson und Bushman (2001) zum Zusammenhang von Gewaltspielnutzung und aggressiven Affekten und Kognitionen

\begin{tabular}{lcccc}
\hline Konstrukt & $\begin{array}{c}\text { Durch- } \\
\text { schnittliche } \\
\text { Korrelation }\end{array}$ & $\begin{array}{c}\text { 95\% } \\
\text { C.I. }\end{array}$ & $\begin{array}{c}\text { Anzahl der } \\
\text { Studien }\end{array}$ & $\begin{array}{c}\text { Anzahl der } \\
\text { Probanden }\end{array}$ \\
\hline $\begin{array}{l}\text { Aggressive Kognitio- } \\
\text { nen }\end{array}$ & .27 & $.22-.31$ & 20 & 1.495 \\
Aggressive Affekte & .18 & $.12-.24$ & 17 & 1.151 \\
\hline
\end{tabular}

Anderson (2004) führte eine Erweiterung und Aktualisierung der bestehenden Metaanalyse von Anderson und Bushman (2001) durch (vgl. Abbildung 10).

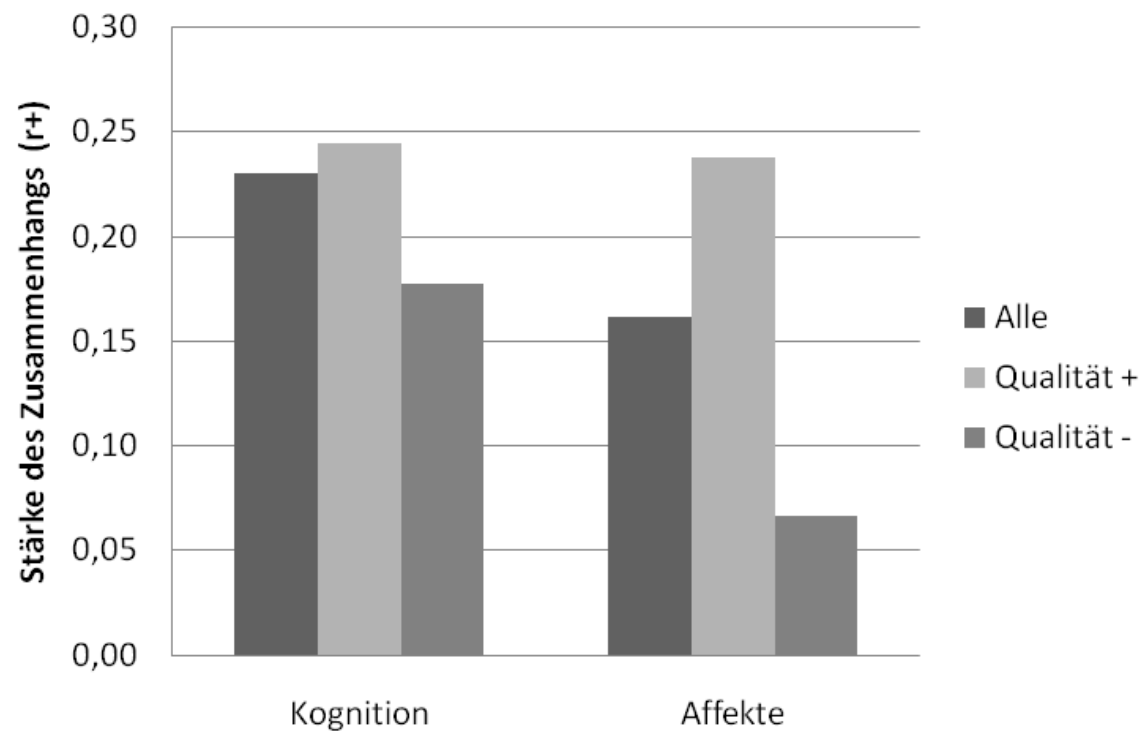

Abbildung 10. Befunde der Metaanalyse von Anderson (2004) zum Zusammenhang von Gewaltspielnutzung und aggressiven Affekten und Kognitionen

In dieser Untersuchung wurde zudem nach den bereits erwähnten Qualitätskennzeichen (vgl. Kapitel 1.3.3.1) zwischen methodisch einwandfreien und methodisch problematischen Studien unterschieden. Insgesamt gingen 19 Studien zu aggressiven Kognitionen und 19 Studien zu aggressiven Affekten in die Analyse ein. Als methodisch einwandfrei eingestufte Untersuchungen ermitteln gerade hinsichtlich der Affekte deutlich höhere positive Zusammenhänge. Auch bezüglich 
aggressiver Kognitionen fällt der Zusammenhang in diesen Untersuchungen höher aus. Insgesamt ergeben sich für beide Konstrukte etwas geringere positive Zusammenhänge mit Gewaltspielnutzung als in der Metaanalyse von 2001. Die Ergebnisse fallen jedoch weiterhin eindeutig aus.

Kurzfristige Wirkungen auf aggressive Kognitionen und Affekte beschränken sich jedoch nicht auf Computerspiele, sondern konnten auch für andere gewalthaltige Medien nachgewiesen werden. Bushman und Huesmann (2006) führten eine Metaanalyse an 431 bis zum Jahr 2000 erschienenen Studien zu den Wirkungen des gesamten Spektrums gewalthaltiger Medien durch. Studien wurden dann eingeschlossen, wenn sie eine Messung oder Manipulation von medialer Gewalt (z. B. Filme, Computerspiele, Musik, Comics) sowie eine Messung von aggressivem Verhalten, prosozialem Verhalten, aggressiven Gedanken, aggressiven Gefühlen oder Arousal beinhalteten. Im Folgenden werden nur die Befunde zu den kurzfristigen Wirkungen auf State-Aggressionsparameter aufgeführt. Hier ergaben sich in ähnlicher Weise positive Zusammenhänge zwischen der Nutzung von Mediengewalt und aggressiven Gefühlen sowie aggressiven Gedanken (vgl. Tabelle 5). Eine differenzielle Auswertung verschiedener Medien, die beispielsweise eine Aussage darüber zulassen würde inwieweit Computerspiele oder Filme ein höheres Wirkpotential aufweisen könnten, wurde in der Studie leider nicht durchgeführt.

Tabelle 5. Befunde der Metaanalyse von Bushman und Huesmann (2006) zum Zusammenhang von Gewaltmediennutzung und aggressiven Affekten und Kognitionen

\begin{tabular}{lcccc}
\hline Konstrukt & $\begin{array}{c}\text { Durch- } \\
\text { schnittliche } \\
\text { Korrelation }\end{array}$ & 95\% C.I. & $\begin{array}{c}\text { Anzahl der } \\
\text { Studien }\end{array}$ & $\begin{array}{c}\text { Anzahl der } \\
\text { Probanden }\end{array}$ \\
\hline $\begin{array}{l}\text { Aggressive Kogni- } \\
\text { tionen }\end{array}$ & .18 & $.17-.19$ & 140 & 22.967 \\
Aggressive Affekte & .27 & $.24-.30$ & 50 & 4.838 \\
\hline
\end{tabular}

Ähnlich wie hinsichtlich des Arousals hat die Annahme kurzfristiger Wirkungen gewalthaltiger Computerspiele und anderer gewalthaltiger Medien auf aggressive Kognitionen und Affekte damit eine empirische Bewährung erfahren. Ein Mangel an Erkenntnissen besteht hier jedoch, ähnlich wie beim Arousal, zu der Frage, wie lange diese kurzfristigen Beeinflussungen anhalten. 
Obwohl der Annahme von Angstreaktionen in Folge medialer Gewaltrezeption eine hohe Augenscheinvalidität zugesprochen werden kann, liegen hierzu vergleichsweise wenige Untersuchungen vor (vgl. Cantor, 2001, S. 207). Zudem widmen sich die meisten Untersuchungen der Fernseh- und Filmgewalt; Untersuchungen zur angstauslösenden Wirkung von gewalthaltigen Computerspielen existieren kaum. Deshalb sollen in diesem Kapitel im Schwerpunkt Studien zur Filmrezeption herangezogen werden um zu klären, wie sich belastende fiktionale Medieninhalte im Allgemeinen auf Angstreaktionen auswirken können.

Es bestehen zunächst Hinweise aus Querschnittsuntersuchungen, dass mediale Gewalt insbesondere im Kindesalter mit Angstreaktionen in Zusammenhang steht: In einer US-amerikanischen Befragungsstudie mit $\mathrm{N}=495$ Eltern von Kindern zwischen 4 und 10 Jahren berichten 8,8 Prozent der Eltern, dass ihre Kinder allwöchentlich Albträume aufgrund des Fernsehens erleben (Owens, et al., 1999). Gentile und Walsh führten eine US-nationalrepräsentative Befragung mit $\mathrm{N}=527$ Eltern mit Kindern im Alter zwischen 2 und 17 Jahren zum innerfamiliären Umgang mit medialen und nicht-medialen Freizeitbeschäftigungsweisen durch. 62 Prozent der Eltern gaben an, dass ihre Kinder Ängste dahingehend erlebt hatten, dass Ereignisse aus dem Fernsehen ihnen selbst zustoßen könnten (Gentile \& Walsh, 2002). Johnson (1980) befragte erwachsene Personen, ob sie schon einmal durch einen Film in besonderer Weise verstört worden seien. Die Teilnehmer wurden gebeten, zu diesem Film anzugeben, wie lange und intensiv sie daraufhin Symptome wie Nervosität, Ängste, Depression oder wiederkehrende Gedanken und Bilder über den Film erlebt hatten. Dabei ergab sich, dass 48 Prozent der Befragten in der Folge der Rezeption mindestens zweitägige intensive Stressreaktionen erlebt hatten. In einer aktuelleren Studie von Harrison und Cantor (1999) gaben von retrospektiv zu einem besonders verstörenden Rezeptionsereignis befragten Collegestudenten 52 Prozent an, dass das Medienereignis Ess- und Schlafgewohnheiten beeinflusst habe und 35 Prozent, dass es zu einem Vermeidungsverhalten hinsichtlich des angstauslösenden Reizes geführt habe (z. B. Vermeidung im Meer zu baden nach der Rezeption eines Films mit Haifischangriffen). Mehr als 60 Prozent gaben an, dass die psychischen Effekte des angstauslösenden Medienereignisses mehr als eine Woche angehalten hatten, etwa 25 Prozent sogar, dass sie das Ereignis immer noch von Zeit zu Zeit beschäftige, obgleich es bereits im Schnitt 6 Jahre zurückgelegen hatte. Das am häufigsten genannte verstörende Medienereignis waren Darstellungen von Blut, Verletzungsfolgen, Schmerzen und andere Formen physischer Beeinträchtigungen gegenüber Lebewesen (Harrison \& Cantor, 1999). 
Es stellt sich die Frage, ob derartige Reaktionen maßgeblich auf das frühe Kindesalter beschränkt sind. Hierbei weist die Studienlage eher darauf hin, dass sich vornehmlich die Angstobjekte ändern, nicht aber die Tatsache, dass Angstreaktionen in Folge von Medienrezeption erlebt werden (vgl. Harrison \& Cantor, 1999). So werden im frühen Kindesalter noch eher Tiere, Dunkelheit, Monster und bestimmte Fabelwesen als beängstigend erlebt, in der späten Kindheit konkretisieren sich die Ängste eher auf die realistischen Folgen von Gewalt und Schädigung wie Verletzungen und Tod und nehmen im späteren Lebensalter auch abstraktere Dimensionen wie etwa Ängste vor Weltproblemen und Kriegen ein (vgl. Cantor, 2001, S. 211 ff.). Dennoch kann davon ausgegangen werden, dass die Reizintensität von medialen Gewaltereignissen im Erwachsenenalter bzw. unter Personen mit habituell erhöhter Gewaltmediennutzung besonders hoch ausfallen muss, um noch eine besondere angstauslösende Wirkung entfalten zu können, was auf den zunehmenden Erwerb kognitiver Copingstrategien (Cantor, 2001) im Umgang mit medialer Gewalt sowie auf Desensibilisierungsprozesse (van der Molen \& Bushman, 2008) zurückgeführt werden kann. Dies bedeutet zum einen, dass sich der Anteil von angstauslösender Gewalt im höheren Lebensalter reduzieren könnte, zum anderen aber auch, dass Personen ihr Medienmenu in der Weise anpassen könnten, dass auch weiterhin angststimulierende Wirkungen erlebt werden (beispielsweise durch den Konsum immer härterer Horror- oder Actionfilme). Weiterhin muss bedacht werden, dass Heranwachsende regelmäßig und über einen längeren Zeitraum der angstauslösenden Wirkung von gewalthaltigen Medien ausgesetzt sein könnten, indem durch das sich alterstypisch verändernde Medienmenu und die entwicklungsbedingten Sensibilitäten immer wieder neue Formen von angstauslösenden Reizen erlebt werden, die über bisherige Rezeptionserfahrungen hinausgehen und dadurch entsprechende Anpassungsreaktionen notwendig machen. ${ }^{40}$

Inwieweit die Ergebnisse der Forschung zur angstauslösenden Wirkung von Film- und Fernsehgewalt auf gewalthaltige Computerspiele zu übertragen sind, ist angesichts der vorhandenen Forschungslage nicht zuverlässig einzuschätzen. Cantor geht von einer besonderen Wirkung gewalthaltiger Spiele aus:

"'Virtual reality' systems take this process even further, resulting in experiences that seem astonishingly similar to undergoing a real event. It seems only logical

40 So zeigen z. B. Vorschulkinder intensive Angstreaktionen auf mediale Ereignisse, denen in späteren Lebensjahren in der Regel keinerlei angstauslösende Bedeutung mehr zukommt (z. B. die Verwandlung von Dr. Bruce Banner in das grüne Monster Hulk). Auf der anderen Seite besitzen Vorschulkinder noch nicht die kognitive Reife, um sich vor einem Film wie "The Day After" zu fürchten, da sie das abstrakte Bedrohungsszenario eines Atomkrieges noch nicht erfassen können. Diese Beispiele können veranschaulichen, dass jede Entwicklungsstufe ihre ganz speziellen Sensibilitäten und damit verbundenen Risiken für spezifische durch Medien verursachte Angstreaktionen bereithalten könnte (vgl. Cantor, 2001). 
that these advances will make scary images all the more frightening to children" (Cantor, 2001, S. 218).

In einer belgischen Studie konnte gezeigt werden, dass bei Albträumen in Zusammenhang mit Fernsehen und Computerspielen insbesondere im frühen Jugendalter noch das Fernsehen dominiert und im späteren Jugendalter beide Medien gleichauf liegen. So ergaben sich bei Jungen $(M[$ Alter] $=13.6, S D=.43)$ mindestens wöchentliche Albträume zum Fernsehen bei elf Prozent und zum Computerspielen bei sechs Prozent. Im Jugendalter $(M[$ Alter] $=16.4, S D=.71)$ lagen mindestens wöchentliche Albträume zum Fernsehen bei drei Prozent, zum Computerspielen bei rund vier Prozent (van den Bulck, 2004 a). Nur eine experimentelle Studie konnte zu der Frage aufgefunden werden, wie sich gewalthaltige Computerspiele auf die situative Ängstlichkeit auswirken. Hierbei handelt es sich um die bereits behandelte Between-Subject-Studie von Baldaro und Kollegen (2004) mit N = 22 männlichen Studienteilnehmern (vgl. Tabelle 3). In diesem Experiment wurde auch eine Präsowie eine Postmessung von situativer Ängstlichkeit (State-Trait Anxiety Inventory-Y) durchgeführt. Ein Anstieg der rezeptionsbedingten Ängstlichkeit zeigte sich nur in der 20-minütigen Gewaltspielbedingung, nicht jedoch in der 20-minütigen gewaltneutralen Spielbedingung. Dieser Befund erscheint bemerkenswert, da es sich bei der Probandengruppe um männliche, erwachsene Studienteilnehmer (Alter: 20 - 29 Jahre) handelte, die zudem seit mehr als fünf Jahren Computerspiele nutzten und dabei mehr als eine Stunde täglich spielten. Ein Anstieg der Feindseligkeit beim Spielen des gewalthaltigen Spiels ergab sich hier hingegen nicht (Baldaro, et al., 2004). Dies zeigt auf, dass hinsichtlich gewalthaltiger Computerspiele dringend weitere Studien erforderlich sind, um das Wirkpotential dieses Mediums auf verschiedene affektive Parameter besser einschätzen zu können.

Hinsichtlich der diskutierten Moderatoren der Angstreaktionen auf mediale Gewalt wird neben dem Lebensalter und damit korrelierenden Gewaltexpositionserfahrungen auch das Geschlecht diskutiert. Insgesamt scheinen Angstreaktionen auf gewalthaltige Medien bei Mädchen zumindest hinsichtlich fiktionaler Inhalte etwas stärker ausgeprägt zu sein (vgl. van der Molen \& Bushman, 2008). Dieser Unterschied zeigt sich jedoch insbesondere in höherem Lebensalter und im Rahmen von Selbsteinschätzungsmaßen der Angstreaktion, die durch Angaben nach sozialer Erwünschtheit (beim männlichen Geschlecht) verzerrt sein könnten (Cantor, 2001, S. 216). Zudem könnten höhere Angstreaktionen bei Mädchen auch dadurch zu erklären sein, dass sie in der Regel geringere Gewaltexpositionserfahrungen aufweisen und damit noch stärker auf gewaltmediale Reize reagieren könnten. 


\subsection{Positive Gefühle (Unterhaltungserleben)}

Die Frage, warum viele Menschen gewalthaltige Medien freiwillig nutzen und das Rezeptionsgeschehen nicht abbrechen, obwohl sie dabei starke Erregungen und negativ valente Emotionen erleben mögen, hat einen ganzen Forschungszweig der Unterhaltungsforschung begründet (Möller, 2006, S. 112). Die Vielzahl der hierzu vorliegenden theoretischen Ansätze können an dieser Stelle unmöglich umfassend behandelt werden (für einen Überblick vgl. Kunczik \& Zipfel, 2004, S. 193 ff.). ${ }^{41}$ Dennoch muss die Annahme eines Stresserlebens in Zusammenhang mit gewalthaltigen Unterhaltungsmedien auch in eine Theorie des Unterhaltungserlebens eingebettet werden, da davon ausgegangen werden sollte, dass innerhalb eines normalen Rezeptionsgeschehens aversiven Gefühlen auch positive Erlebnisaspekte gegenüberstehen müssen.

Gewalthaltige Filme und Computerspiele werden oftmals von ihren Nutzern als besonders "spannend" erlebt. Zur Erklärung dieser mit einem Anspannungsempfinden verbundenen Form positiv erlebter Unterhaltung hat in besonderer Weise die Theorie der Erregungsübertragung von Zillmann beigetragen, die auch auf gewalthaltige Computerspiele übertragen werden kann (vgl. Klimmt, 2004). In einem Film entsteht Spannung in der Regel dadurch, dass zunächst sympathische Figuren (Protagonisten) eingeführt werden, mit denen sich der Zuschauer identifiziert und die dann in eine bedrohliche Lage geraten, bei der der Zuschauer sich nicht sicher sein kann, ob die Geschichte ein gutes Ende nehmen wird (vgl. Zillmann, 2003, S. 117). Die Bedrohungen können dabei je nach Film und Genre verschiedene Formen annehmen (z. B. ein Angriff von Zombies in einem Horrorfilm oder die Übernahme eines Flughafens durch Terroristen in einem Actionfilm). Für den Zuschauer entsteht nun die Motivation, der Geschichte unbedingt weiter folgen zu wollen um zu sehen, ob die Protagonisten die Bedrohung erfolgreich abwehren können. Die hiermit in Zusammenhang stehende Anspannung kann auch in fiktionalen Formaten sehr hoch ausfallen:

"Ungeachtet der Übertragung von durch Fiktion hervorgerufenen Emotionen in das reale Leben ist die Gewährleistung von emotionalen Achterbahnfahrten jedoch eines der primären Ziele der Unterhaltung; und es können kaum Zweifel daran bestehen, dass es den Unterhaltungsmedien gelingt, unsere Emotionen zu manipulieren und mit ihnen zu spielen. Häufig erzeugen sie dabei Affektstärken, die der Angst gegenüber wirklichen persönlichen Herausforderungen

41 Viele dieser Motive sind zudem allgemeine Nutzungsmotive von Unterhaltungsmedien bzw. Computerspielen, wie die Überbrückung von Langeweile, strukturelle Kopplung zwischen Medieninhalt und Lebenswirklichkeit (parallel oder kompensatorisch), Eskapismus und Flowerleben. 
oder dem Hochgefühl nach deren Bewältigung nahe kommen und oft sogar gleichkommen" (Zillmann, 2003, S. 120).

Diese Bedrohungssituationen werden in der Regel am Ende der Filmrezeption durch ein gutes Ende (Happy End) wieder aufgelöst. Nach der Theorie der Erregungsübertragung bleiben die im Rahmen der Rezeption erlebten Anspannungsgefühle jedoch noch über die Rezeptionssituation hinaus bestehen und werden bei einem gutem Ausgang kognitiv umgedeutet und als positive Gefühle bzw. Euphorie erlebt. Bei gewalthaltigen Computerspielen ergibt sich eine ähnliche Situation: Hier schlüpft der Spieler in die Rolle eines Avatars (einer Spielfigur), der ebenfalls einer bedrohlichen Situation bzw. feindseligen Umgebung ausgesetzt ist, in der sich dieser in der Regel heldenhaft bewähren muss. Hierbei verläuft das Anspannungserleben nun weniger über Identifikationsprozesse mit einem oder mehreren Protagonisten, sondern vielmehr aus der Ungewissheit, ob der Spieler selbst in der Rolle des Protagonisten der virtuellen Bedrohungssituation entkommen bzw. die Feinde/Gegner erfolgreich besiegen kann. ${ }^{42}$ Klimmt (2004, S. 9) nimmt an, dass die Erregungsübertragung bei Computerspielen noch stärker als bei Filmen ausfallen müsste, weil der Spieler den positiven Ausgang als die eigene Rettung und nicht über den Umweg der Identifizierung als die Rettung eines oder mehrerer geliebter Protagonisten erlebt.

Für Zillmann kann die Theorie der Erregungsübertragung auf alle von Medien ausgelösten Emotionen angewendet werden und auch erklären, warum Menschen mit dem Einsatz von Medien ihre Stimmungen effektiv regulieren und verändern können (Mood Management). Dabei kann gerade das wechselseitige Erleben von unangenehmen und angenehmen Emotionen ein intensives Unterhaltungserleben charakterisieren und Stimmungszustände verändern. Diese theoretische Einbettung des positiven Arousalpotentials von gewalthaltigen Computerspielen kann damit veranschaulichen, warum die generelle Zuwendungsattraktivität von physiologischen Reaktionen nicht betroffen sein muss und damit ein rezeptionsbedingtes Erleben von Stress und negativen Gefühlen nicht in Widerspruch zum Unterhaltungsanspruch gewalthaltiger Medien steht.

Hieraus lässt sich zudem die Annahme ableiten, dass emotionale Beanspruchung in Folge der Mediennutzung nicht zwangsläufig auch subjektiv als solche wahrgenommen werden muss. In einer Studie der Forschungsgruppe Psychophysiologie des Instituts für Psychologie der Universität Freiburg wurden an N = 100 Kindern im Alter von 11 und $\mathrm{N}=100$ Jugendlichen im Alter von 15 Jahren Herzfrequenzund Bewegungsmessungen über einen 23-stündigen Zeitraum durchgeführt (Myrtek, 2003; Myrtek \& Scharff, 2000). Die emotionale Beanspruchung der Kinder

42 Hierauf bauen weitere Unterhaltungstheorien zum Computerspielen auf wie das Erleben von Selbstwirksamkeit und Flowerleben (vgl. Klimmt, 2004S. 9 ff.). 
wurde zum einen physiologisch erfasst, indem die Herzfrequenz gemessen und dabei auch die tatsächliche motorische Aktivität berücksichtigt wurde, zum anderen in Form von Selbstberichten in 15-minütigen Intervallen erhoben. Hier gaben die Teilnehmer auch an, mit welcher Aktivität sie aktuell beschäftigt waren. Bemerkenswerterweise zeigte sich, dass die Heranwachsenden ihre Beanspruchung in der Schule subjektiv als höher einschätzten als jene während des Fernsehens, die physiologischen Daten jedoch gegenteilig anzeigten, dass während des Fernsehens eine größere emotionale Beanspruchung vorherrschend war als während der Schulzeit.

\subsubsection{Zusammenfassung}

Die Sichtung des Forschungsstandes zu kurzfristigen Wirkungen gewalthaltiger Computerspiele auf affektive und kognitive Parameter lässt sich wie folgt zusammenfassen:

1. Computerspielrezeption ist ein aktiver Vorgang, der eine Vielzahl von kognitiven Prozessen beanspruchen kann. Förderliche Aspekte zeigen sich insbesondere hinsichtlich räumlich-visueller und motorischer Leistungen. Der Forschungszweig zu Wirkungen von Computerspielen auf die kognitive Leistungsfähigkeit hat jedoch bislang die Frage nach dem möglicherweise differenziellen Wirkpotential gewalthaltiger vs. gewaltneutraler Computerspiele weitestgehend ausgeblendet. Zudem stützen sich die Studien nahezu exklusiv auf Förderungstheorien, indem betrachtet wird, ob die bei Computerspielnutzung trainierten maßgeblich räumlich-visuellen Fertigkeiten zu einer Verbesserung eben dieser Fertigkeiten beitragen können. Eine breitere Betrachtung der kognitiven Wirkungen unterschiedlicher Computerspielformate, die mögliche "Trade-Offs" zwischen verschiedenen Leistungskomponenten (vgl. Greenfield, 2009) berücksichtigt und damit eine Beurteilung der Forschungsdaten hinsichtlich relevanter Transfereffekte auf realweltliche Leistungskontexte, etwa in der Schule, erlauben würde, ist bislang nur in Ansätzen erkennbar.

2. Das affektive Wirkpotential gewalthaltiger Computerspiele manifestiert sich in einer erhöhten physiologischen Erregung, die sowohl durch kardiovaskuläre als auch katecholamine Stressreaktionen gekennzeichnet ist. Dabei zeigt sich in nahezu allen Untersuchungen, dass gewalthaltige Spiele stärkere Reaktionen hervorrufen als gewaltneutrale Spiele. Stärkere physiologische Reaktionen konnten zudem auch im Vergleich gewalthaltiger und gewaltmoderater Spiele aufgefunden werden (Brady \& Matthews, 2006). Auch konnte gezeigt werden, dass erhöhtes Arousal bei Computerspielen möglicherweise besonders begünstigt wird, wenn diese von Blut- und Splattereffekten begleitet werden (Ballard 
\& Wiest, 1996) oder besonders realistische Gewalt beinhalten (Barlett \& Rodeheffer, 2009). Dieses Befundmuster wird zusätzlich dadurch gestützt, dass Gewaltereignisse in gewalthaltigen Spielen offenbar unmittelbar mit negativem Gefühlserleben im Rahmen erhöhten Arousals korrelieren (Ravaja, et al., 2008; Salminen \& Ravaja, 2008). Die Frage, ob gewalthaltige Computerspiele dabei eine höhere physiologische Erregung begünstigen als gewalthaltige Filme ist erst wenig untersucht worden. Theoretische Erwägungen sprechen für diese Annahme. Die einzige hierzu bislang vorhandene Studie deutet hinsichtlich kardiovaskulärer Erregungsparameter auf eine höhere Wirkung gewalthaltiger Spiele hin (Maass, et al., 2010).

3. Es zeigt sich, dass physiologische Reaktionen auch über die Rezeptionssituation hinaus wirken können (Carnagey, et al., 2007; Frindte \& Obwexer, 2003). Unterschiede zwischen gewalthaltigen und gewaltneutralen Medien zeigen sich hier jedoch kaum (Carnagey, et al., 2007). Nach der Theorie der Erregungsübertragung von Zillmann (vgl. 2003) können durch Medien ausgelöste Gefühlsreaktionen noch weit über die Rezeption hinaus bestehen bleiben und das postrezeptive Aktivierungsniveau sowie die emotionale Reaktivität erhöhen.

4. Die im Rahmen experimenteller Untersuchungen festgestellte Erhöhung von State-Aggressivität und aggressiven Kognitionen gibt zusätzlich Aufschluss darüber, dass gewalthaltigen Computerspielen ein hohes Wirkpotential auf emotionale Erlebniszustände zugesprochen werden kann. Obgleich hinsichtlich des die Rezeption gewalthaltiger Computerspiele begleitenden emotionalen Wirkspektrums von einer hohen Komplexität und interindividuellen Variabilität ausgegangen werden muss und hierzu noch gravierende Erkenntnislücken bestehen, sollen die affektiven Wirkungen gewalthaltiger Spiele in dieser Arbeit im Rahmen eines Stressparadigmas interpretiert werden. Es wird im weiteren Verlauf der Frage nachgegangen, inwieweit sich gewalthaltige Computerspiele als psychische Stressoren auffassen lassen, denen ähnliche kognitive Wirkungen zugesprochen werden können wie anderen bekannten relativen Stressoren. Diesbezüglich wird im folgenden Kapitel der Frage nachgegangen, wie sich das durch gewalthaltige Computerspiele induzierte Stresserleben auf explizite Gedächtnisfunktionen auswirken könnte. 


\subsection{Die Bedeutung von Arousal und Stress für die semantische Gedächtnisleistung}

\subsubsection{Kurzüberblick über die Systematik des Gedächtnisses}

Während "Lernen" den Prozess beschreibt, bei dem es zu einem Erwerb von Wissensbeständen kommt, beschreibt der Begriff "Gedächtnis" die diesem Prozess zugrundliegenden Fähigkeiten eines Organismus zur Enkodierung und Konsolidierung von Informationen sowie ihrem späteren Abruf (Kandel, Kupfermann, \& Iversen, 2000, S. 1227). Dabei wird zwischen einem expliziten (deklarativen) und einem impliziten Gedächtnis unterschieden. Das implizite Gedächtnis kennzeichnet Gedächtnisleistungen, die nicht bewusstseinsfähig sind, wie zum Beispiel das Priming, das prozedurale Gedächtnis, das assoziative Lernen (Konditionierung) und das nicht-assoziative Lernen wie Habituation. Explizite Gedächtnisleistungen sind hingegen bewusstseins- und auskunftsfähig. Das explizite Gedächtnis umfasst das episodische Gedächtnis, in welchem autobiographische Informationen abgelegt werden, das Wissens- bzw. Faktengedächtnis (z. B. Schulwissen), sowie das Bekanntheits- oder Vertrautheitsgedächtnis (Kandel, et al., 2000; Roth, 2001, S. 151 ff.). Für diese Untersuchung ist das explizite Wissens- bzw. Faktengedächtnis von maßgeblicher Bedeutung. ${ }^{43}$

Hinsichtlich des Erwerbs expliziter Wissensbestände sind drei besonders relevante Funktionen zu unterscheiden (Kandel, et al., 2000, S. 1237 ff.; Wolf, 2009, S. 144). Die erste Funktionseinheit ist die Enkodierung (Encoding), die sowohl die Aufmerksamkeitsfokussierung zu Informationen als auch deren Weiterverarbeitung umfasst. Die Qualität der Enkodierung und der Grad, mit dem die neuen Wissensbestände mit bereits bestehenden Wissensbeständen in Beziehung gebracht werden, wird als entscheidend dafür erachtet, wie effektiv die Informationen später abgerufen und in aktuelle Problemlöseprozesse einbezogen werden können. Die zweite Funktionseinheit ist die Konsolidierung (Consolidation) der Informationen. Auf zellulärer Ebene betrachtet basiert diese auf der Optimierung von Informationen in den synaptischen Verbindungen neuronaler Netzwerke (Parkin, 2000, S. 26). Zum Teil wird von der Konsolidierung auch noch die Funktionseinheit Speicherung (Storage) und damit der Erhalt von Informationen abgegrenzt, die

43 Vor diesem Hintergrund sind die kognitiven Wirkungen von Computerspielen maßgeblich als das Resultat impliziter Lernvorgänge zu verstehen (vgl. Kapitel 1.3.2). So sind die positiven kognitiven Wirkungen von Computerspielen im Sinne einer Verbesserung räumlichvisueller Fähigkeiten, computerspielbezogener Regeln und Strategien sowie motorischer Fähigkeiten (Handaugenkoordination) ebenso dem impliziten Gedächtnissystem zuzuordnen wie negative kognitive Wirkungen, etwa das kurzfristige Priming aggressiver Kognitionen und eine langfristige Desensibilisierung für mediale Gewalt (Habituation). 